OPEN ACCESS

Edited by:

Hasan Ayaz,

Drexel University, United States

Reviewed by:

Brent Winslow,

Design Interactive, United States

Solaiman Shokur,

Alberto Santos Dumont Association for Research Support, Brazil

*Correspondence: Ronak Patel

ronak.patel@imperial.ac.uk

Specialty section:

This article was submitted to

Neural Technology,

a section of the journal

Frontiers in Neuroscience

Received: 27 August 2019

Accepted: 28 October 2019

Published: 15 November 2019

Citation:

Patel R, Ashcroft J, Patel A Ashrafian $\mathrm{H}$, Woods $A J$, Singh $\mathrm{H}$, Darzi A and Leff DR (2019) The Impact

of Transcranial Direct Current

Stimulation on Upper-Limb Motor

Performance in Healthy Adults: A

Systematic Review and Meta-Analysis.

Front. Neurosci. 13:1213

doi: 10.3389/fnins.2019.01213

\section{The Impact of Transcranial Direct Current Stimulation on Upper-Limb Motor Performance in Healthy Adults: A Systematic Review and Meta-Analysis}

\author{
Ronak Patel ${ }^{1 *}$, James Ashcroft ${ }^{1}$, Ashish Patel ${ }^{1}$, Hutan Ashrafian ${ }^{1}$, Adam J. Woods ${ }^{2}$, \\ Harsimrat Singh ${ }^{1}$, Ara Darzi ${ }^{1}$ and Daniel Richard Leff ${ }^{1}$ \\ ${ }^{1}$ Department of Surgery \& Cancer, Imperial College London, London, United Kingdom, ${ }^{2}$ Department of Clinical and Health \\ Psychology, Center for Cognitive Aging and Memory, McKnight Brain Institute, University of Florida, Gainesville, FL, \\ United States
}

Background: Transcranial direct current stimulation (tDCS) has previously been reported to improve facets of upper limb motor performance such as accuracy and strength. However, the magnitude of motor performance improvement has not been reviewed by contemporaneous systematic review or meta-analysis of sham vs. active tDCS.

Objective: To systematically review and meta-analyse the existing evidence regarding the benefits of tDCS on upper limb motor performance in healthy adults.

Methods: A systematic search was conducted to obtain relevant articles from three databases (MEDLINE, EMBASE, and PsycINFO) yielding 3,200 abstracts. Following independent assessment by two reviewers, a total of 86 articles were included for review, of which 37 were deemed suitable for meta-analysis.

Results: Meta-analyses were performed for four outcome measures, namely: reaction time $(R T)$, execution time $(E T)$, time to task failure (TTF), and force. Further qualitative review was performed for accuracy and error. Statistically significant improvements in RT (effect size $-0.01 ; 95 \% \mathrm{Cl}-0.02$ to $0.001, p=0.03$ ) and ET (effect size $-0.03 ; 95 \% \mathrm{Cl}$ -0.05 to $-0.01, p=0.017$ ) were demonstrated compared to sham. In exercise tasks, increased force (effect size $0.10 ; 95 \% \mathrm{Cl} 0.08$ to $0.13, p<0.001$ ) and a trend towards improved TTF was also observed.

Conclusions: This meta-analysis provides evidence attesting to the impact of tDCS on upper limb motor performance in healthy adults. Improved performance is demonstrable in reaction time, task completion time, elbow flexion tasks and accuracy. Considerable heterogeneity exists amongst the literature, further confirming the need for a standardised approach to reporting tDCS studies.

Keywords: transcranial direct-current stimulation (tDCS), systematic review, meta-analysis, motor, healthy, performance 


\section{INTRODUCTION}

Transcranial Direct Current Stimulation (tDCS) is a noninvasive method of brain stimulation proposed to have beneficial effects in both cognitive and motor domains. Benefits have been demonstrated in patients with chronic pain syndromes (Fregni et al., 2006; Fenton et al., 2009; Fagerlund et al., 2015) and neuropsychiatric conditions (Baker et al., 2010; Loo et al., 2012; Palm et al., 2012; Kaski et al., 2014; Bandeira et al., 2016; Breitling et al., 2016), whilst in the healthy population, there is increasing scientific interest in the motor enhancing properties of the technology. Aligning with this trend, an increasing number of commercial companies (Edwards et al., 2017) promote the augmentation of motor abilities with tDCS including greater muscular power output (Okano et al., 2015; Huang et al., 2019), longer athletic endurance (Vitor-Costa et al., 2015; Park et al., 2019) and improved posture and balance (Kaminski et al., 2016; Saruco et al., 2017). This arena is most commonly explored through anodal tDCS to the primary motor cortex (M1), although the precise mechanism of action remains a matter of debate (Giordano et al., 2017). Excitability changes within M1 have been demonstrated, as evidenced through an increase in size of motor evoked potentials within the small muscles of the hand (Nitsche and Paulus, 2000, 2001). Similarly, tDCS transiently modulates cortical activation by raising the resting membrane potential of neurons closer to the activation threshold, thus increasing neuronal excitability (Bindman et al., 1964; Nitsche and Paulus, 2000). These neurophysiological changes persist after stimulation and are suggested to be associated with upregulation in N-methyl-d-aspartate receptor activation (Liebetanz et al., 2002). Regardless of these neurophysiological findings, there is a lack of consensus on the impact of tDCS on motor function in healthy individuals.

Despite a recent surge in meta-analyses on the effect of tDCS on aspects of cognitive function (Medina and Cason, 2017; Nilsson et al., 2017; Westwood and Romani, 2017; Simonsmeier et al., 2018), efforts to quantify the impact on motor function in healthy individuals are few in number (Bastani and Jaberzadeh, 2012; Hashemirad et al., 2016; Machado et al., 2019). Notably, Bastani and Jaberzadeh (2012) performed a metaanalysis focusing on motor cortex excitability and motor function but only included two studies involving healthy participants. Subsequently, Hashemirad et al. (2016) observed that multiple tDCS sessions over M1 induced significant task improvement but this review was limited to motor sequence learning. Other narrative reviews have summarized the effects of tDCS on motor tasks in healthy individuals with enhancing effects demonstrated in bimanual motor skills (Pixa and Pollok, 2018), motor learning (Reis and Fritsch, 2011; Buch et al., 2017), and exercise performance (Angius et al., 2017).

Whilst prior reviews (Reis and Fritsch, 2011; Angius et al., 2017; Buch et al., 2017; Pixa and Pollok, 2018) provide valuable summaries of tDCS studies, a meta-analysis would confer more critical and robust assessment of the impact of tDCS on motor function. Firstly, meta-analysis better estimates the effects that exist within the target population rather than limited to individual studies. Secondly, precision and accuracy of effect sizes is improved through pooled data offering greater statistical power than smaller separate sample sizes. Furthermore, it facilitates identification of methodological patterns or variables that could contribute to conclusions or, similarly, identify inconsistencies that lead to discrepancies within findings.

To date, there has been no systematic evaluation and metaanalysis of the overall impact of tDCS on upper limb motor performance in healthy adults and this paper aims to provide an up-to-date comprehensive analysis of available literature in this regard.

\section{METHODS}

\section{Search Strategy}

A comprehensive electronic search (Appendix 1), of three databases was conducted, namely: (a) MEDLINE (1946-August 2018), (b) PsycINFO (1806-August 2018), and (c) EMBASE (1947-August 2018). Due to variability in motor tasks and outcomes in tDCS literature, the search initially identified all randomised-controlled trials involving tDCS. Additional studies were gathered from cross-referencing bibliographies of included papers and from Google Scholar. The date of the last search conducted was 01 August 2018.

\section{Eligibility Criteria}

Retrieved articles were only included if they met the following inclusion criteria:

1. Studies performed on healthy subjects.

2. Studies requiring subjects to perform a motor task involving the upper limbs

3. Studies with published outcome variable data (raw or summary statistics)

4. Sham-controlled studies.

Reviews, case reports, letters, opinions, and conference abstracts were not included. Studies were limited to those carried out on adult human subjects and reported in English language. Any studies using subjects with prior expertise in tasks were not included e.g., pianists in finger tapping tasks or strengthtrained athletes in elbow flexion tasks. Any studies which utilized additional interventions alongside tDCS, including pharmacological or other neuro-interventions (e.g., Transcranial Magnetic Stimulation), were also excluded.

\section{Data Extraction}

Titles and abstracts of all retrieved articles were screened by three of the reviewers (RP, JA, and AP) to identify relevant studies. Relevant articles that met inclusion criteria were obtained in full text and further assessed for eligibility by the same authors. Any disagreements during the selection process were resolved by discussion with a fourth, senior author (HA). Final selected studies are summarized in Table $\mathbf{1}$.

A data extraction form was generated in Microsoft Excel for Mac Version 16.19 (Microsoft Corporation, Redmond, WA, USA), and the following data were recorded: author, sample size, anode/cathode location, current intensity, experimental task, and performance outcome measure. Where possible, the first motor 
TABLE 1 | Characteristics of studies selected for pooled statistical analysis.



R, right; L, left; $A+C$, anodal and cathodal montages used; M1, Primary Motor Cortex; C-SOR, Contralateral Supraorbital Region; SMA, Supplementary Motor Area; PMC, Pre-motor Cortex; PFC, Prefrontal Cortex; RT, reaction time; ET, execution time; TTF, time to failure; MIVC, maximal isometric voluntary contraction; 1 RM, 1 repetition maximum; JHFT, Jebsen Hand Function Test. 
assessment following the first single session of stimulation was used as the post-stimulation measurement. Moreover, significant efforts were made to obtain relevant missing data. Specifically, 19 authors were emailed to request further data, of which six responded.

\section{Quality and Risk of Bias Assessment}

Three bias assessment tools were employed to ensure robust evaluation. The quality and the risk of bias of selected articles were independently assessed by two authors (RP and JA). Quality was assessed using the Jadad score (Jadad et al., 1996) and the van Tulder scale (van Tulder et al., 2003). The Cochrane risk of bias tool (Higgins and Green, 2011) was additionally applied to RCTs with assessment of its seven key components. Any disagreement regarding quality or bias assessment was resolved through discussion with a senior author (HA).

\section{Data Analysis}

Outcome measures including reaction time, task completion time, time to failure, and force, were identified to allow statistical pooling of results. For each outcome measure, individual meta-analyses were performed using all relevant data sources regardless of stimulation protocol. However, where comparative studies used a variety of stimulation sites, further subgroup analyses were performed to examine the change in effect size using only anodal motor cortex stimulation (with variable cathodal placement). Pooled incidence and outcome measures were calculated through a random effects model employing an inverse variance Der Simonian Laird meta-analytical methodology (Tan et al., 2016). Study heterogeneity was appraised through the $I^{2}$ statistic and meta-analysis was performed in Microsoft Excel for Mac Version 16.19 (Microsoft Corporation, Redmond, WA, USA) and Stata Version 15 (Stata Corp LP, College




Station, TX, USA). Where meta-analysis was not possible, narrative review was performed for additional evaluation of relevant literature.

\section{RESULTS}

\section{Selected Articles}

The flow of articles through the selection process is depicted in Figure 1. Following de-duplication, the literature search yielded 3,200 articles. Following exclusions, 86 relevant articles remained for detailed review. Articles were then subcategorized based on availability of performance outcome data suitable for pooled meta-analysis. These included the following outcome variables: reaction time (RT), execution time (ET), time to task failure (TTF), and force in muscle strength tasks. In total, 37 articles remained for final meta-analysis.

\section{Overview of Literature}

A total of 86 articles yielded 184 individual montage experiments investigating the impact of tDCS on upper limb motor tasks and there was demonstrable methodological heterogeneity amongst these, as illustrated in Figure 2. The typical stimulation protocol utilized $1 \mathrm{~mA}$ with $35 \mathrm{~cm}^{2}$ electrodes pads delivering a currently density of $0.029 \mathrm{~mA} / \mathrm{cm}^{2}$ (30\%). Of the total, 43\% $(n=79)$ applied stimulation for $20 \mathrm{~min}$ and $70 \%(n=130)$ used an online approach with motor tasks carried out during the stimulation period. As further illustrated in Figure 2C, motor cortex stimulation was the most frequent target area of choice (67\%). There was variability with regard to the montage arrangement within each target area. During motor stimulation, the supraorbital region was the most common (67\%) location for the reference electrode.

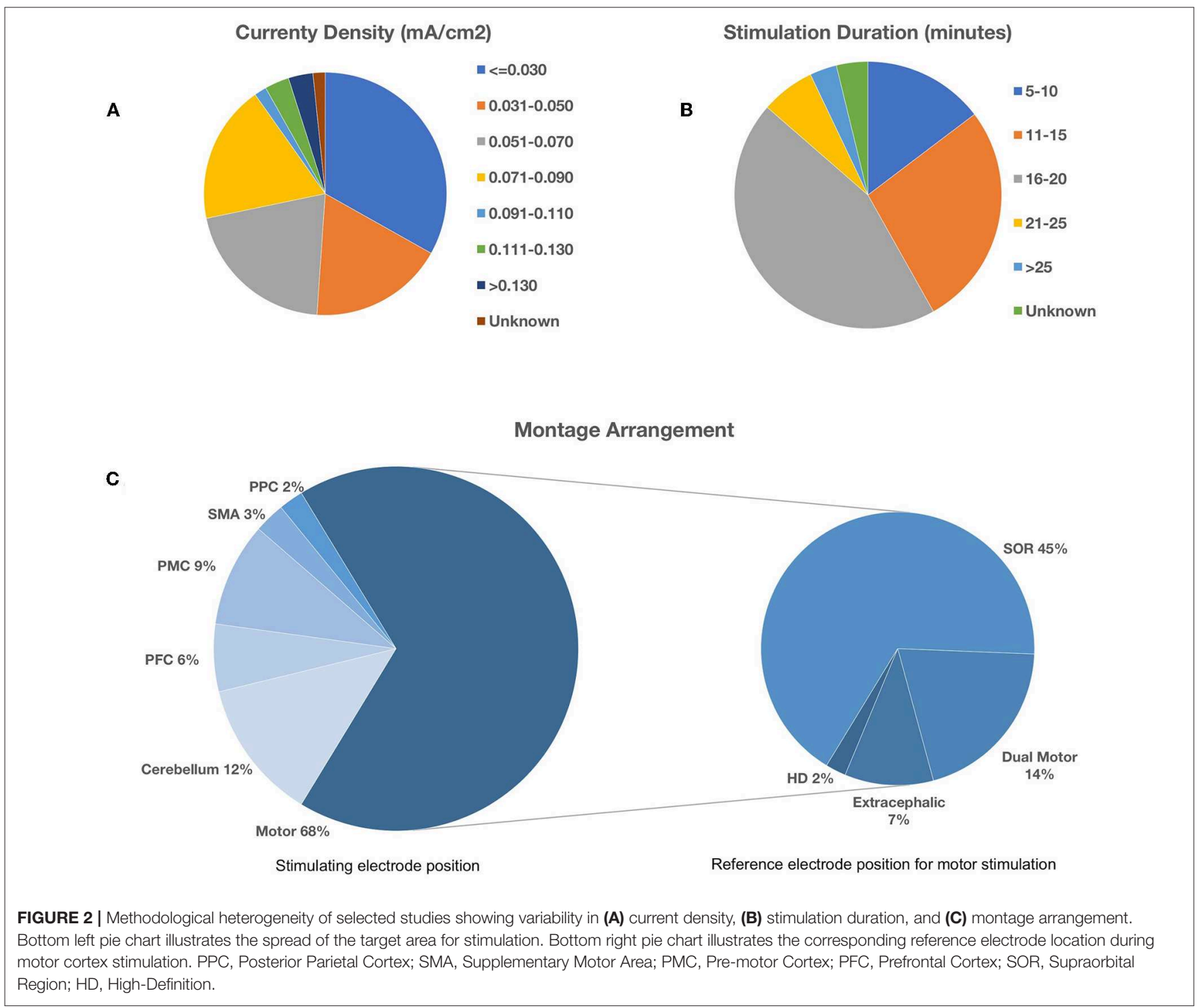




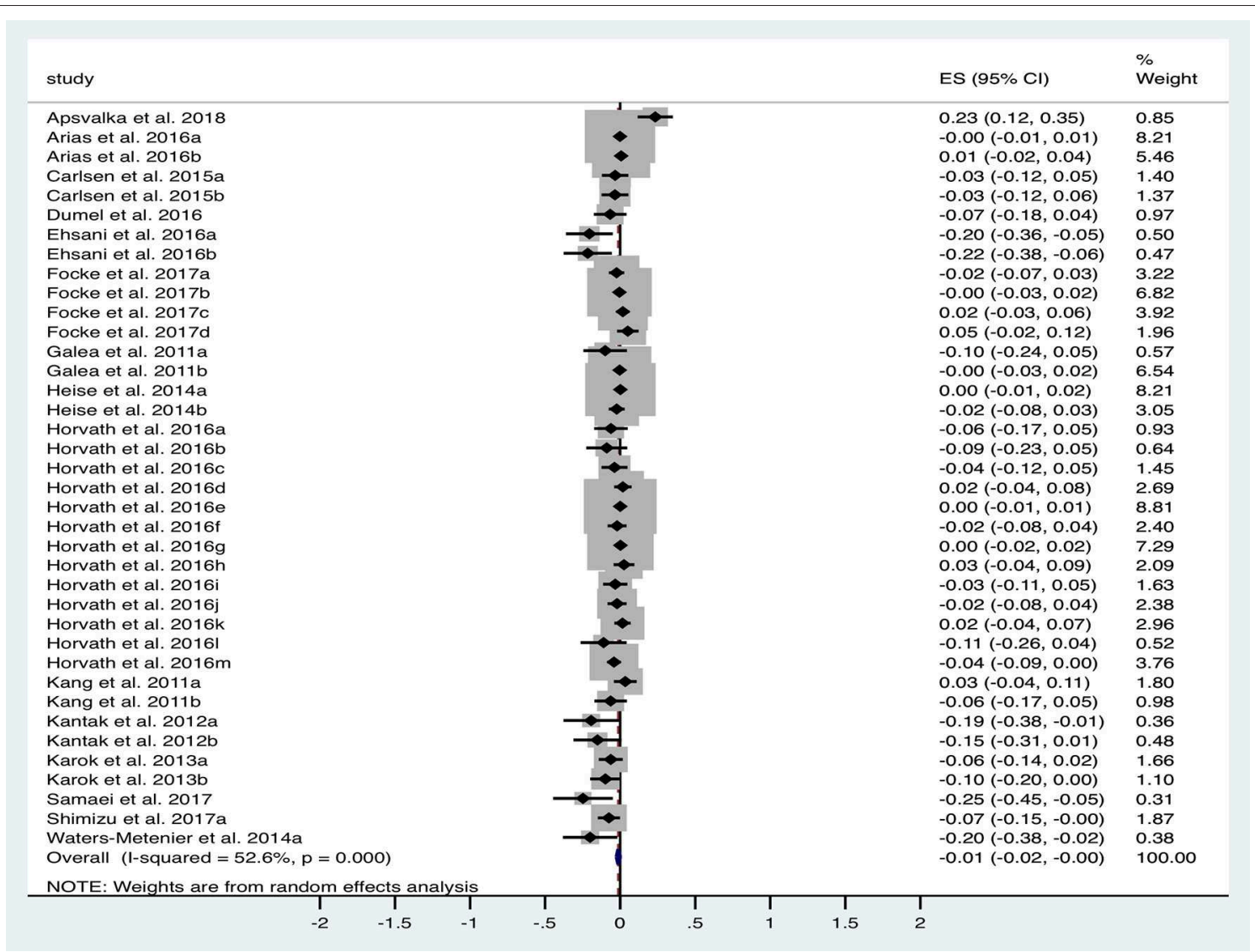

FIGURE 3 | Forest Plot illustrating effect sizes from the comparison in reaction time between tDCS vs. sham. Positive values indicate an increase in reaction time following anodal tDCS whilst negative values indicate a decrease in reaction time. Grey boxes represent the weight given to each study. Error bars represent $95 \%$ confidence intervals.

TABLE 2 | Stimulation protocols and outcomes of additional studies investigating the effect of tDCS on reaction time in an upper limb motor task.

\begin{tabular}{|c|c|c|c|c|c|c|c|c|}
\hline References & $\begin{array}{l}\text { Sample } \\
\text { size }\end{array}$ & Stimulation & Reference & $\begin{array}{c}\text { Current } \\
(\mathrm{mA})\end{array}$ & $\begin{array}{l}\text { Current density } \\
\left(\mathrm{mA} / \mathrm{cm}^{2}\right)\end{array}$ & $\begin{array}{c}\text { Duration } \\
\text { (min) }\end{array}$ & Task & Significant effect vs. Sham \\
\hline Ambrus et al. (2016) & 17 & L M1 $(A+C)$ & C-SOR & 1 & 0.029 & $12-14$ & SRTT & $\mathrm{Nil}$ \\
\hline Ferrucci et al. (2013) & 21 & Cerebellum & R arm & 2 & 0.057 & 20 & SRTT & $\uparrow$ \\
\hline Herzfeld et al. (2014) & 51 & L M1; Cerebellum $(A+C)$ & $\begin{array}{l}\text { C-SOR; } \\
\text { R Buccinator }\end{array}$ & 2 & 0.080 & 25 & Hand reaching & Nil \\
\hline Lindenberg et al. (2013) & 20 & L M1 & C-SOR; R M1 & 1 & 0.029 & 30 & Choice RTT & $\mathrm{Nil}$ \\
\hline Lindenberg et al. (2016) & 24 & L M1 & C-SOR; R M1 & 1 & 0.029 & 30 & $\mathrm{RTT}$ & $\mathrm{Nil}$ \\
\hline Nitsche et al. (2003b) & 80 & $\begin{array}{l}\text { L M1; PMC; L lateral } \\
\text { PFC; } \\
\text { L medial PFC (all A+C) }\end{array}$ & C-SOR; R M1 & 1 & 0.029 & 15 & SRTT & $\uparrow$ in L M1 \\
\hline Nitsche et al. (2010) & 44 & LPMC $(A+C)$ & C-SOR & 1 & 0.029 & 15 & SFTT; SRTT & $\begin{array}{l}\uparrow \text { with } A \text { stimulation } \\
\text { in } R E M \text { sleep }\end{array}$ \\
\hline
\end{tabular}

Stimulation sites are anodal unless otherwise specified. R, right; L, left; A, anodal, C, cathodal; M1, Primary Motor Cortex; C-SOR, Contralateral Supraorbital Region; PMC, Pre-motor Cortex; PFC, Prefrontal Cortex; DLPFC, Dorsolateral Prefrontal Cortex; SRTT, Serial Reaction Time Task; SFIT, Serial Finger Tapping Task; $\uparrow$, denotes improvement in performance with stimulation; $\downarrow$, denotes worse performance with stimulation; Nil, no significant effect of tDCS on performance compared to sham stimulation. 


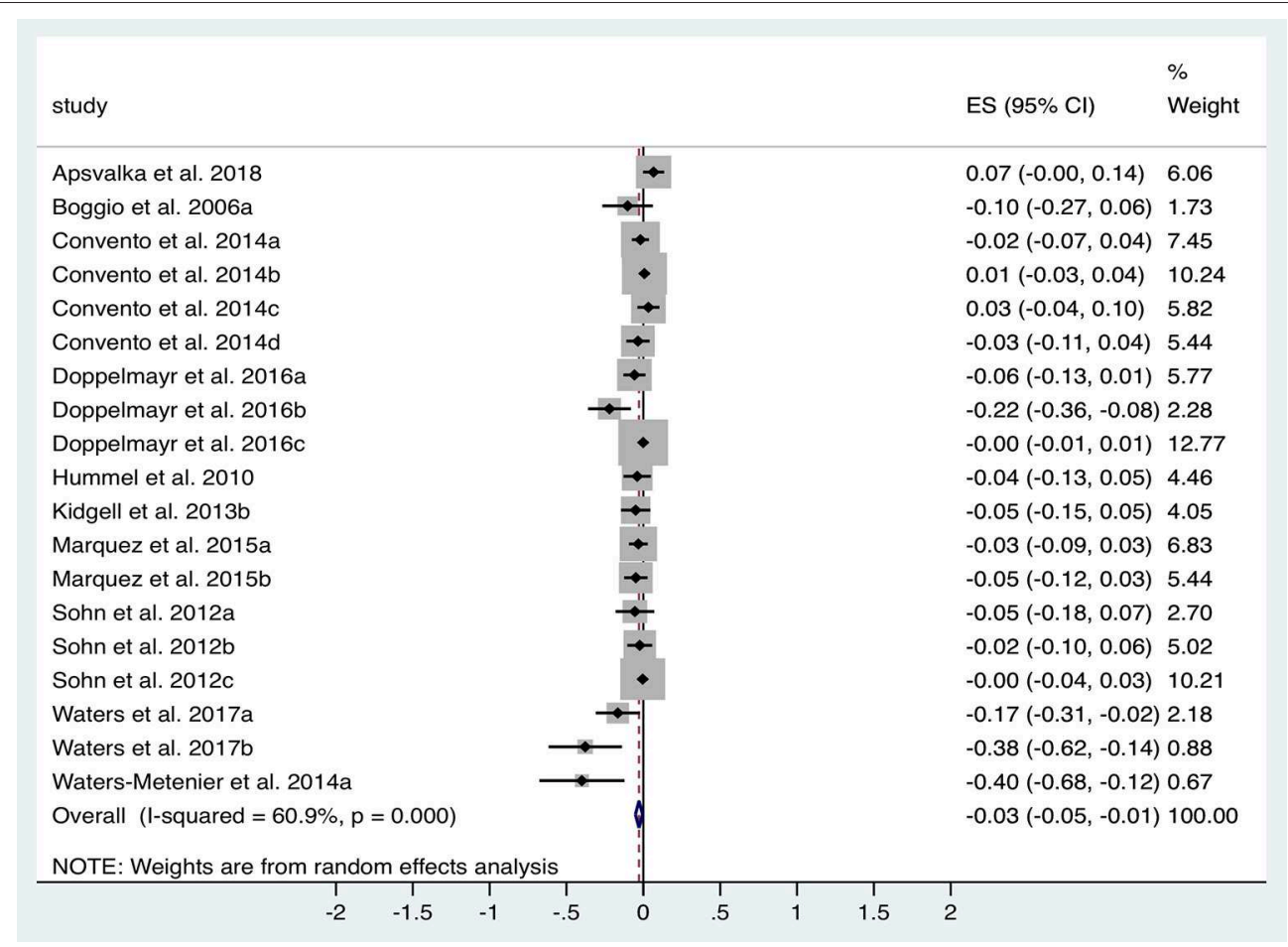

FIGURE 4 | Forest Plot illustrating effect sizes from the comparison in total task time between tDCS vs. sham. Positive values indicate an increase in time taken following anodal tDCS whilst negative values indicate a decrease in time taken. Grey boxes represent the weight given to each study. Error bars represent $95 \%$ confidence intervals.

\section{Upper Limb Dexterity Tasks-Reaction Time}

A total of 15 studies ( $n=618$ subjects) were suitable for quantitative analysis of the effect of tDCS vs. sham on RT. As illustrated in Figure 3, tDCS significantly reduced RT, albeit with a small effect size (ES $0.01,95 \%$ CI -0.02 to $0.001, p=$ 0.03). Significant heterogeneity was observed when comparing tDCS to sham $\left(I^{2}=53 \% ; \chi^{2}=78.09, p<0.001\right)$. Subgroup analysis of anodal motor stimulation did not alter these results (ES $-0.01,95 \% \mathrm{CI}-0.03$ to $-0.00, p=0.049$ ). Additional within-group analyses for tDCS and sham groups did not achieve statistical significance. Numerous other studies (summarized in Table 2) investigated the impact of tDCS on RT in a motor task but could not be included in the meta-analysis due to a lack of published raw data. Of these studies, 50\% reported improvement with tDCS ( $80 \%$ motor stimulation), which is consistent with the observed marginally beneficial statistical effect size.

\section{Upper Limb Dexterity Tasks-Execution Time}

A total of 10 studies ( $n=344$ subjects) were suitable for analysis of the impact of tDCS vs. sham on ET. Figure 4 illustrates the significant reduction in time taken to complete dexterity tasks following tDCS compared to sham with an effect size of -0.03 (95\% CI -0.05 to $-0.01, p=0.017$ ). Significant heterogeneity was observed $\left(I^{2}=61 \% ; \chi^{2}=46.03\right.$, $p<0.001)$. Subgroup analysis of anodal motor montages marginally increased the effect size to -0.04 (95\% CI -0.07 to $-0.01, p=0.002$ ).

Additional within-group analyses was performed on 11 studies for both tDCS and sham compared to baseline. Overall effect size for tDCS was -0.09 (95\% CI -0.13 to $-0.05, p<0.001)$ compared to -0.03 (95\% CI -0.05 to $-0.004, p=0.02)$ for sham. Subgroup analysis of anodal motor stimulation confirmed these results for both tDCS (ES -0.09) and in sham (ES -0.02). Additional studies without available data for pooled analysis support overall findings with improved ET in a Purdue Pegboard Test (Karok et al., 2017) and a sport cup stacking task (Pixa et al., 2017a).

\section{Upper Limb Dexterity Tasks-Accuracy/Error}

Numerous studies have explored the impact of tDCS on a series of motor tasks with accuracy and error as outcome measures (Table 3). There is widespread heterogeneity amongst these studies not only in methodological design but also with regard to the task and the definition of the accuracy and error outcome measure. Therefore, we summarize the various montages these and subcategorize them according to the type of outcome measure, namely: correct responses, distance error, degree of error, error count, "skill" (calculated from error 
TABLE 3 | Stimulation protocols and outcomes of studies investigating the effect of tDCS on different accuracy and error measurements in motor tasks.

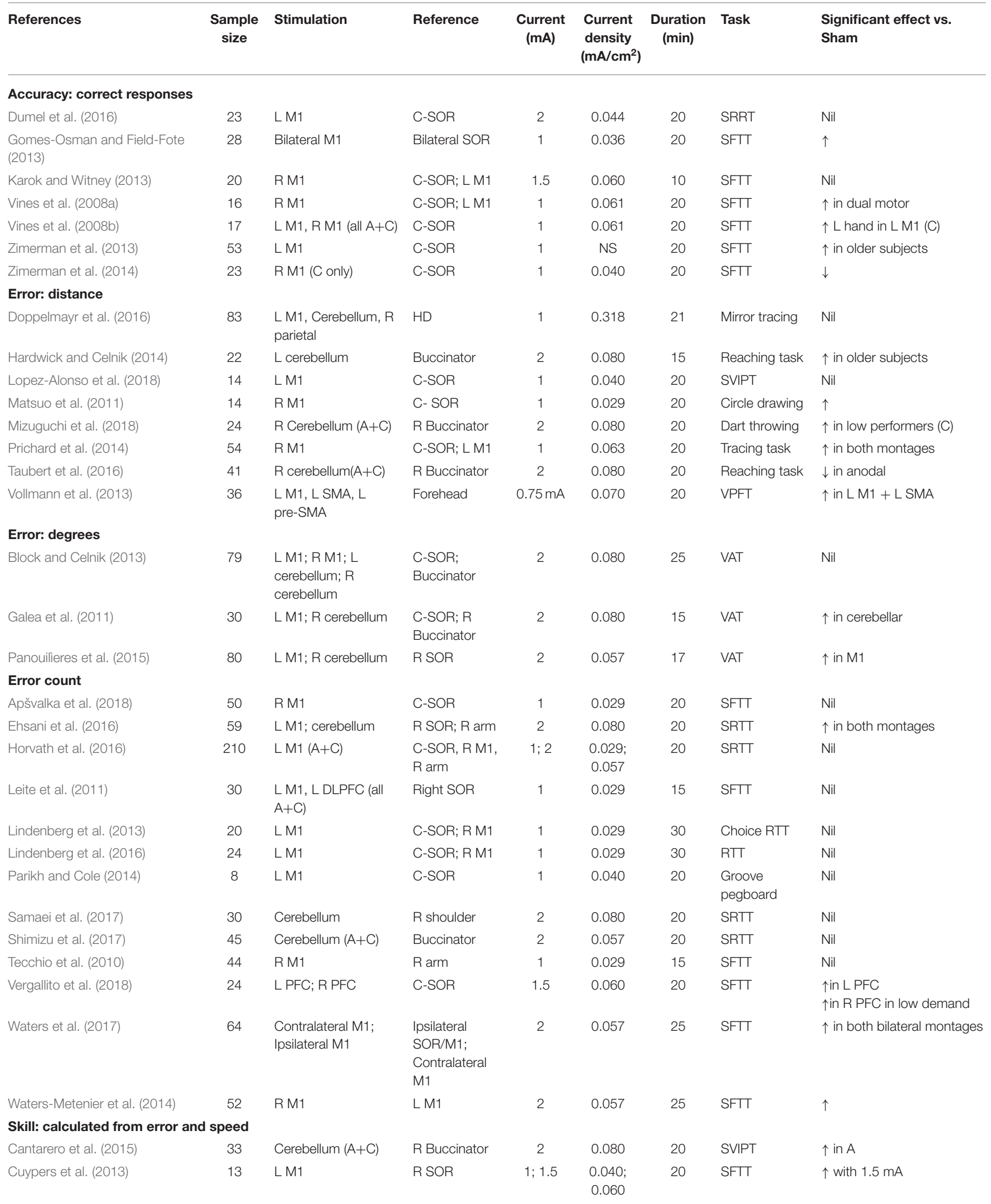


TABLE 3 | Continued

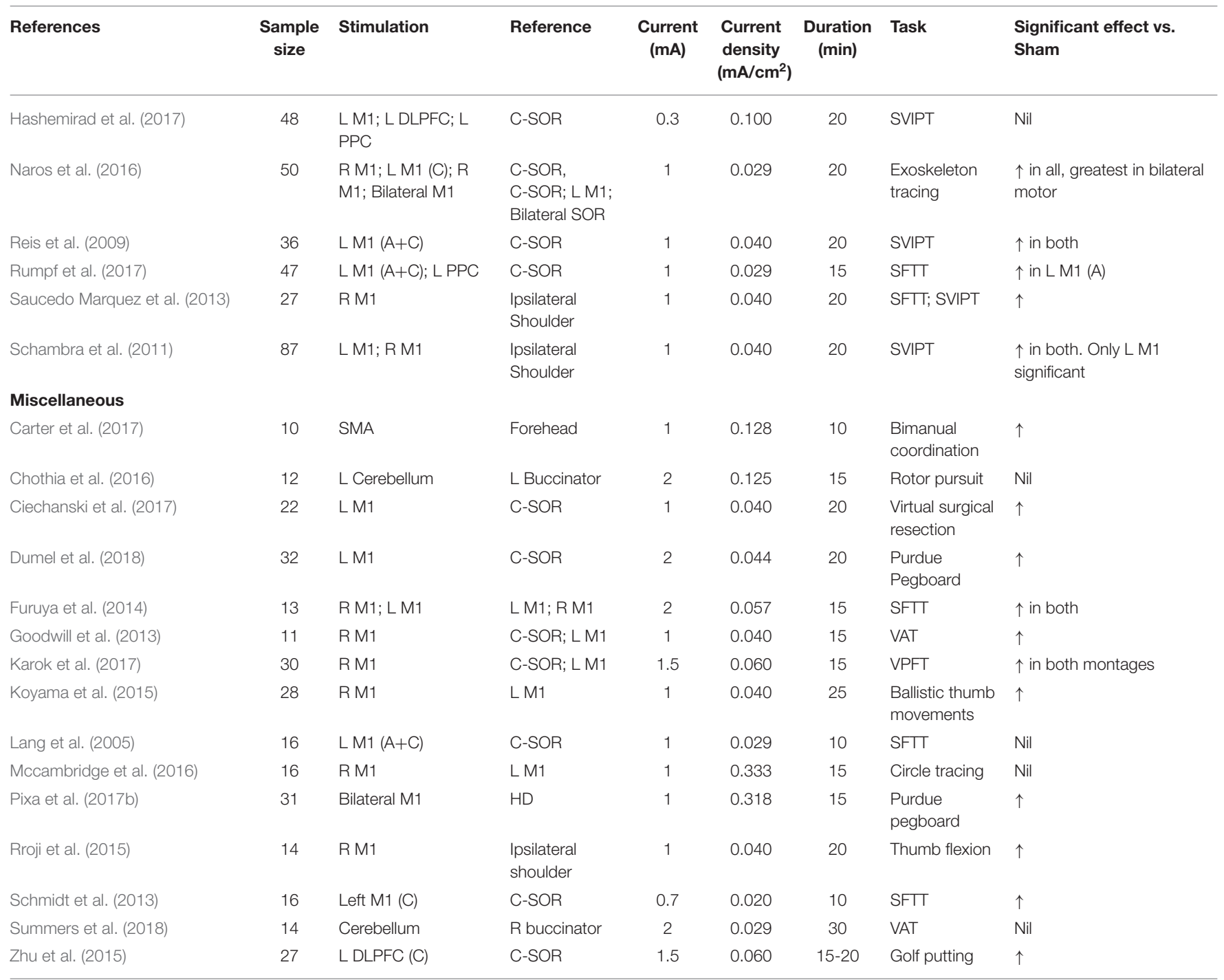

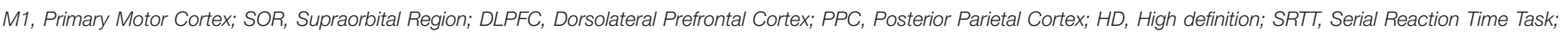

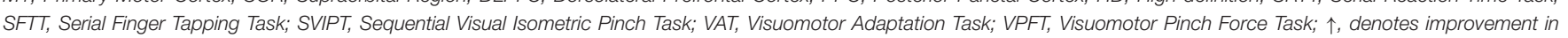
performance with stimulation; $\downarrow$, denotes worse performance with stimulation; Nil, no significant effect of tDCS on performance compared to sham stimulation.

and speed measurements of a motor task) and miscellaneous outcome measures.

Dual (Vines et al., 2008a; Gomes-Osman and Field-Fote, 2013; Karok and Witney, 2013) and unilateral dominant (Zimerman et al., 2013) motor cortex stimulation increased the number of correct responses in a sequential finger tapping task (SFTT), but was not replicated in other studies (Vines et al., 2008b; Dumel et al., 2016). Cathodal stimulation to the non-dominant (Zimerman et al., 2014) motor cortex decreased the number of correct responses in SFTT. tDCS led to improved skill outcomes, in the majority of studies applying motor cortex stimulation (Reis et al., 2009; Schambra et al., 2011; Cuypers et al., 2013; Saucedo Marquez et al., 2013; Naros et al., 2016; Rumpf et al., 2017). Similarly, motor stimulation also demonstrated improvements in a variety of miscellaneous tasks (Table 3). Only cerebellar stimulation in this context failed to confer any improvements in motor performance.

Drawing task distance error improvements were less consistent with benefits in non-dominant and dual (Matsuo et al., 2011; Prichard et al., 2014), but not dominant motor cortex stimulation (Doppelmayr et al., 2016). Other distance error tasks benefitted with motor (Vollmann et al., 2013) and cerebellar (Hardwick and Celnik, 2014; Mizuguchi et al., 2018) stimulation, but not consistently amongst the literature (Taubert et al., 2016; Lopez-Alonso et al., 2018). Although improvements were demonstrated in visuomotor adaptation tasks (error in degrees) with motor (Panouilieres et al., 2015) and cerebellar (Galea et al., 2011) stimulation, this was inconsistent (Galea et al., 2011; Block and Celnik, 2013; Panouilieres et al., 2015). Only a 


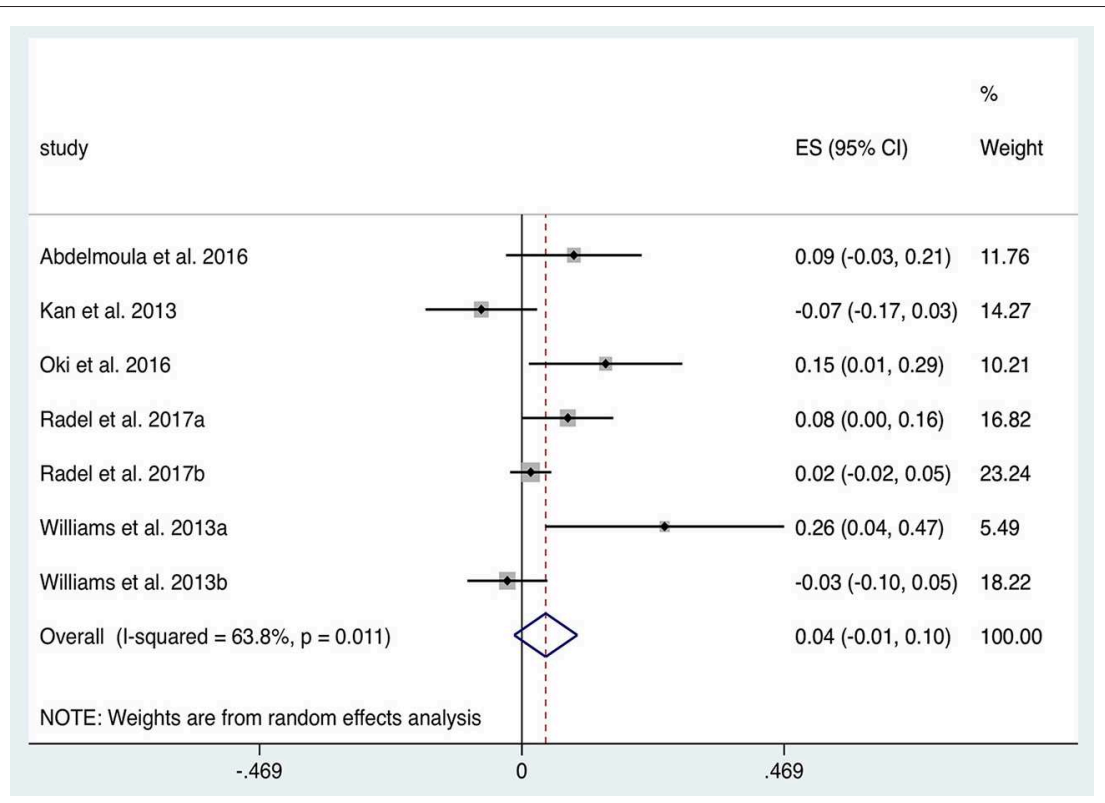

FIGURE 5 | Forest Plot illustrating effect sizes from the comparison in time to elbow flexion task failure between anodal tDCS vs. sham tDCS. Positive values indicate an increase in time to failure following tDCS whilst negative values indicate a decrease in time. Grey boxes represent the weight given to each study. Error bars represent $95 \%$ confidence intervals.

minority of studies (Waters-Metenier et al., 2014; Ehsani et al., 2016; Waters et al., 2017; Vergallito et al., 2018) investigating error count in a SRTT and SFTT demonstrated improved performance with $\mathrm{tDCS}$, all of which had substantial variation in stimulation montages.

\section{Upper Limb Exercise Tasks: Fatigue}

In total five studies with $n=79$ subjects were suitable for quantitative analysis of the effect of tDCS on TTF in elbow flexion tasks. Figure 5 illustrates a tendency towards prolonged TTF with tDCS compared to sham (ES $0.04,95 \%$ CI -0.01 to 0.10 , $p=0.139)$. Heterogeneity was observed when comparing anodal tDCS to sham in this cohort of studies $\left(I^{2}=64 \% ; \chi^{2}=16.59, p\right.$ $=0.01)$. Subgroup analysis of anodal motor montages increased the effect size to 0.06 ( $95 \% \mathrm{CI}-0.04$ to $0.16, p=0.269)$.

\section{Upper Limb Exercise Tasks: Strength}

Studies investigating the impact of tDCS on strength of contraction in upper limb flexion/extension tasks were divided into four studies with a fatiguing contraction between pre- and post- measurements (therefore causing a decrease in strength) and five studies without such a contraction. The five studies without a fatiguing contraction ( $n=73$ subjects) provided data for within-group analysis of change in strength from baseline in tDCS and sham groups. Anodal motor tDCS increased strength (ES $0.10,95 \%$ CI 0.08 to $0.13, p<0.001$; Figure 6A) twice as much as sham (ES 0.05, 95\% CI 0.03 to $0.08, p<$ 0.001 ; Figure 6B). Both of these analyses exhibited significant heterogeneity $(p<0.001)$. A repeated stimulation protocol was utilized in three studies and stimulation was combined alongside strength training (ST) in four studies. An additional study
(Lampropoulou and Nowicky, 2013), not included due to lack of data, showed no effect of tDCS on strength.

Elbow flexion strength was examined either side of a fatiguing contraction in four studies. Within-group analyses revealed similar reductions in strength effect size from baseline in intervention (ES $-0.26,95 \% \mathrm{CI}-0.32$ to $-0.19, p<0.001$ ) and sham groups (ES $-0.22,95 \% \mathrm{CI}-0.28$ to $-0.17, p<0.001$ ). Subgroup analysis of anodal motor stimulation was comparable.

\section{Quality Scoring and Risk of Bias Assessment}

Summary risk of bias graph is illustrated in Figure 7 and Results of Jadad Score and Van Tulder quality assessment scores are summarized in Table 4. Randomization was utilized in $78 \%$ of studies but only $14 \%$ were deemed to sufficiently explain methods used for random sequence generation. A double-blind approach was used in $65 \%$ of studies with the remaining $16 \%$ reporting only single-blinding and $19 \%$ did not mention blinding at all. Generally, studies performed well in terms of selective reporting, avoiding co-interventions, retaining acceptable compliance and assessing outcomes at similar time-points.

\section{DISCUSSION}

This study provides a comprehensive and contemporaneous review and quantitative analysis of the effect of tDCS on in healthy adults. In regard to dexterity tasks, the present analysis has demonstrated a modest improvement in reaction time and significant improvements in execution time and other performance domains of accuracy and error with tDCS. Analysis of muscle strength studies revealed significant strength 


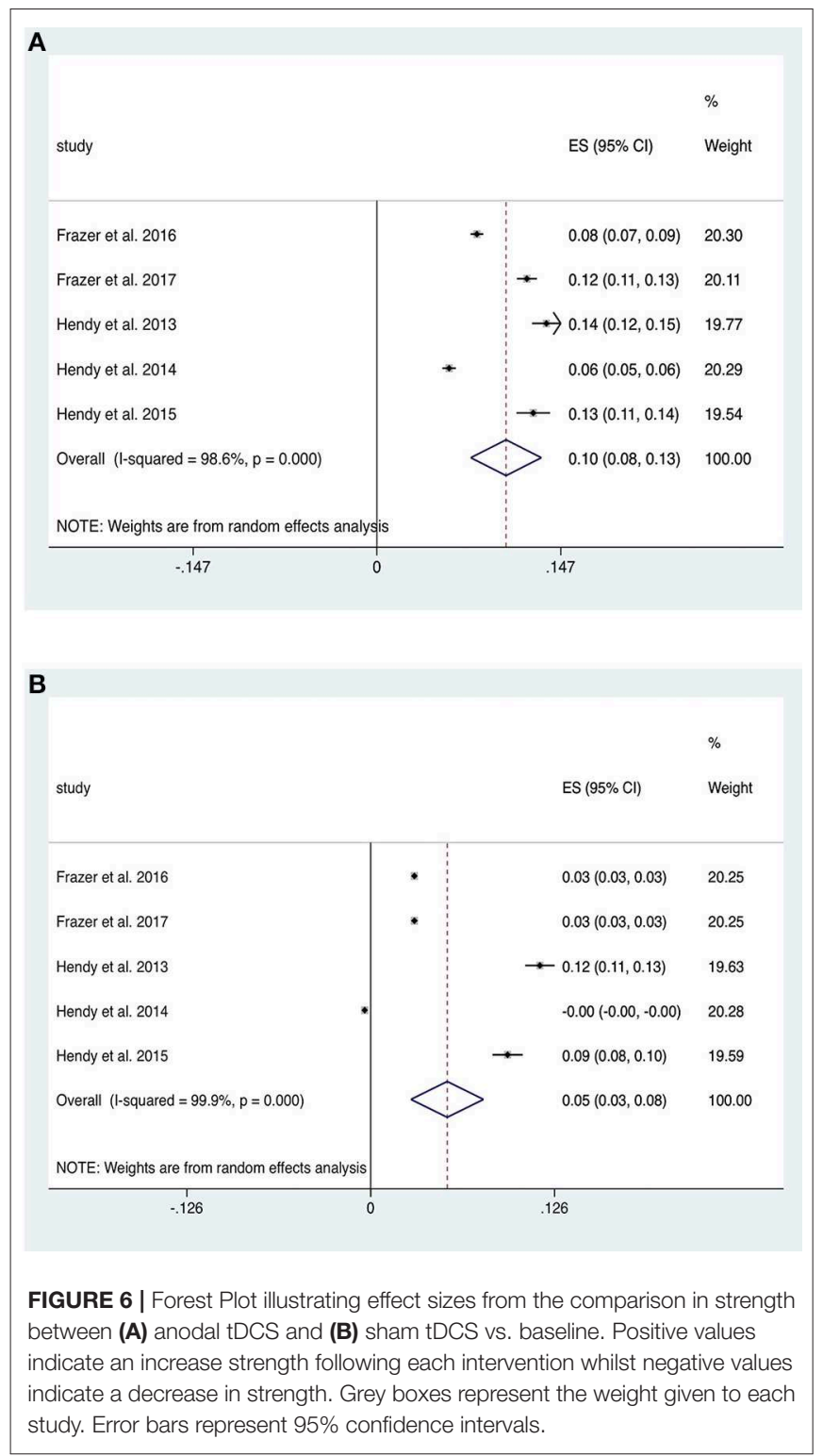

improvement with training along with a tendency towards reduced fatigue with $\mathrm{tDCS}$.

\section{Upper Limb Dexterity Tasks}

Reduction in motor RT is frequently used as a representation of motor learning, and, numerous studies demonstrate significant reduction in reaction time with tDCS compared to sham. This was commonly observed in unilateral (Nitsche et al., 2003b; Kantak et al., 2012; Karok and Witney, 2013; Heise et al., 2014; Dumel et al., 2016, 2018; Ehsani et al., 2016) and dual (Karok and Witney, 2013; Waters-Metenier et al., 2014) anodal motor stimulation or anodal cerebellar stimulation (Ferrucci et al., 2013; Ehsani et al., 2016; Samaei et al., 2017) with benefits consistent at $24 \mathrm{~h}$ retention tests as well (Shimizu et al., 2017). However, improvements were not universal throughout the literature with similar stimulation protocols (Nitsche et al., 2003b; Galea et al.,
2011; Stagg et al., 2011; Lindenberg et al., 2013, 2016; Heise et al., 2014; Ambrus et al., 2016; Arias et al., 2016; Horvath et al., 2016; Focke et al., 2017; Apšvalka et al., 2018). Interestingly, RT worsened with cathodal stimulation regardless of site (Leite et al., 2011; Stagg et al., 2011; Carlsen et al., 2015; Shimizu et al., 2017), potentially due to reduced motor cortex excitability with cathodal tDCS (Nitsche et al., 2003a). Further benefits of tDCS in motor tasks was demonstrable with improvements in ET three times greater than sham, a difference made even more apparent when isolating anodal motor stimulation only. All studies with single session anodal stimulation of the non-dominant motor cortex demonstrated improved performance (Boggio et al., 2006; Williams et al., 2010; Sohn et al., 2012; Kidgell et al., 2013; Convento et al., 2014; Parikh and Cole, 2014; Karok et al., 2017). This was not demonstrated with stimulation of the dominant cortex (Boggio et al., 2006; Sohn et al., 2012; Convento et al., 2014) and it is possible that the comparative lack of observed effect on the dominant hand could be due to a ceiling-effect with little room for improvement. However, it could still be beneficial in this context with motor training (Dumel et al., 2018) or in older adults (Hummel et al., 2010). An additional study (Marquez et al., 2015) demonstrated improved performance of the non-dominant hand regardless of laterality of motor cortex stimulation. Amongst other measures of motor performance in dexterity tasks, there is demonstrable and reliable $(85 \%$ of studies) improvement with dual motor stimulation (Vines et al., 2008a; Gomes-Osman and Field-Fote, 2013; Goodwill et al., 2013; Karok and Witney, 2013; Furuya et al., 2014; Prichard et al., 2014; Waters-Metenier et al., 2014; Koyama et al., 2015; Naros et al., 2016; Karok et al., 2017; Pixa et al., 2017b; Waters et al., 2017). Unilateral motor stimulation was less consistent with as many studies documenting improvement (Matsuo et al., 2011; Reis and Fritsch, 2011; Schambra et al., 2011; Cuypers et al., 2013; Goodwill et al., 2013; Karok and Witney, 2013; Saucedo Marquez et al., 2013; Schmidt et al., 2013; Vollmann et al., 2013; Zimerman et al., 2013; Prichard et al., 2014; Panouilieres et al., 2015; Rroji et al., 2015; Ehsani et al., 2016; Naros et al., 2016; Rumpf et al., 2017; Dumel et al., 2018) as no effect (Lang et al., 2005; Vines et al., 2008a,b; Tecchio et al., 2010; Leite et al., 2011; Block and Celnik, 2013; Lindenberg et al., 2013, 2016; Parikh and Cole, 2014; Doppelmayr et al., 2016; Dumel et al., 2016; Horvath et al., 2016; Hashemirad et al., 2017; Apšvalka et al., 2018; Lopez-Alonso et al., 2018).

\section{Upper Limb Exercise Performance}

A trend towards increased time to task failure (TTF) with anodal tDCS compared to sham, which was demonstrated in both online and offline stimulation protocols of elbow flexion tasks. The impact of offline tDCS between two fatiguing contractions $1 \mathrm{~h}$ apart was examined in three studies (Cogiamanian et al., 2007; Kan et al., 2013; Abdelmoula et al., 2016), two of which (Cogiamanian et al., 2007; Abdelmoula et al., 2016) resulted in improved TTF suggesting potential to help reduce neuromuscular fatigue. Interestingly, all three studies showed no difference between strength (as measured by force) between stimulation and sham. The remaining three studies (Williams et al., 2013; Oki et al., 2016; Radel et al., 2017) utilized an online 


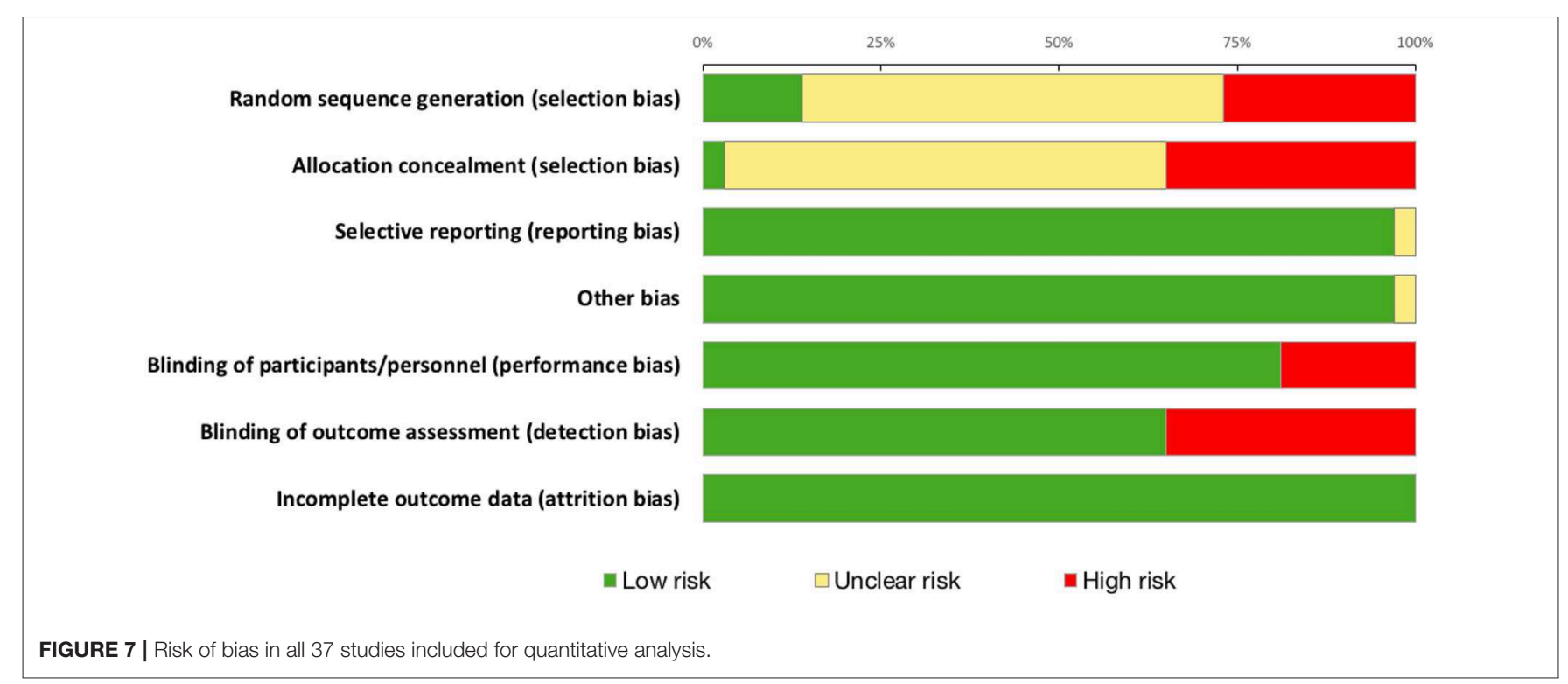

stimulation protocol, two of which (Williams et al., 2013; Oki et al., 2016) demonstrated an improved TTF. Of note, Williams et al. (2013) performed a subgroup analysis which revealed significantly increased TTF in subjects who had stimulation throughout the task against those who had stimulation for part of the task duration. The former was also found to have worsening strength performance. Although overall there seems to be no consistent effect of tDCS on contraction force when separated by a fatiguing contraction, there does appear to be significantly increased force without such contraction. Indeed, tDCS was found to increase by strength twice as much than sham although it must be noted that this is not a direct comparative analysis. Although methodological variability exists within this pool of studies, separate within-group analyses facilitates a robust comparison of tDCS against sham.

These findings align with a recent meta-analysis by Lattari et al. (2018) on effects of tDCS on upper and lower limb muscle strength which demonstrated improved overall improved muscular endurance (TTF) and strength (force of MVC). More recently, Machado et al. (2019) revealed improved TTF with anodal M1 tDCS in cycling but unlike the present study did not analyse TTF in upper limb tasks. They failed to observe an effect of tDCS on strength in upper limb tasks, although they separated isometric, isokinetic and dynamic upper and lower limb exercises and do not report on three studies (Hendy and Kidgell, 2013; Hendy et al., 2015; Frazer et al., 2017) we included. The current analysis further strengthens the case for the potential of tDCS as an ergogenic aid in tasks requiring muscular endurance and strength, with a potentially more profound impact with training and repeated stimulation.

\section{Neural Mechanisms}

The vast majority of electrode montages in these experiments performed motor cortex stimulation. The mechanism underlying motor learning through tDCS has been postulated as a result of increased excitability of the motor cortex augmenting successful and active synaptic connections between the neuronal structures activated by tDCS (Bindman et al., 1964). This is supported by neurophysiological studies which demonstrate the importance of M1 in early learning (Karni et al., 1995) and also consolidation of learning (Ungerleider et al., 2002; Doyon et al., 2009). However, despite the overall trends for improved motor performance, the evidence is inconsistent. There may be several explanations for these divergent findings. Firstly, there is considerable experimental variation with regard to tDCS parameters (stimulation intensity, duration, anode and cathode placement; see Figure 2), experimental design (e.g., online/offline protocols, timing of motor performance, variable washout periods) and motor tasks and their outcome measures. Secondly, with regards to mechanistic effects, some studies have revealed either minimal change or a decrease in M1 excitability (Jenkins et al., 1994; Toni et al., 1998; Floyer-Lea and Matthews, 2005) suggesting that modulation of this area may not be as influential as previously thought, especially given the large influence of other brain structures in facilitating voluntary movement. Similarly, it is maybe a too simplistic a view to suggest that altering M1 excitability alone will impact on motor learning. Given the well-documented roles of other cortical regions and their interconnections (Doyon et al., 2002; Ungerleider et al., 2002; Hardwick et al., 2013) in performing motor skills, it is perhaps unsurprising that there is such variation in the brain region targeted for stimulation with tDCS. Therefore, it is conceivable that to observe significant gains in motor learning tasks, the reliance on other motor brain areas must be accounted for and augmented as well-a notion which may account for our findings of more consistent improvement with dual motor stimulation (see Table 3). Finally, disparate effects of tDCS may be related to the combination of tasks implemented as slight changes in task can not only affect performance, but also learning processes (Nitsche et al., 2003b; Saucedo Marquez et al., 2013).

Underlying neural mechanisms regarding exercise performance are unclear and a number of factors have been 
TABLE 4 | Total Jadad and Van Tulder studies for each study included in quantitative analysis.

\begin{tabular}{|c|c|c|}
\hline References & Jadad score & Van Tulder score \\
\hline Apšvalka et al. (2018) & 1 & 7 \\
\hline Arias et al. (2016) & 1 & 5 \\
\hline Carlsen et al. (2015) & 0 & 6 \\
\hline Dumel et al. (2016) & 1 & 6 \\
\hline Ehsani et al. (2016) & 5 & 10 \\
\hline Focke et al. (2017) & 3 & 9 \\
\hline Galea et al. (2011) & 3 & 9 \\
\hline Heise et al. (2014) & 3 & 8 \\
\hline Horvath et al. (2016) & 1 & 5 \\
\hline Kang and Paik (2011) & 3 & 8 \\
\hline Kantak et al. (2012) & 1 & 5 \\
\hline Karok and Witney (2013) & 2 & 6 \\
\hline Samaei et al. (2017) & 4 & 9 \\
\hline Shimizu et al. (2017) & 1 & 6 \\
\hline Waters-Metenier et al. (2014) & 3 & 8 \\
\hline Boggio et al. (2006) & 4 & 9 \\
\hline Convento et al. (2014) & 3 & 8 \\
\hline Doppelmayr et al. (2016) & 4 & 9 \\
\hline Hummel et al. (2010) & 3 & 7 \\
\hline Karok et al. (2017) & 2 & 6 \\
\hline Kidgell et al. (2013) & 4 & 8 \\
\hline Marquez et al. (2015) & 5 & 10 \\
\hline Parikh and Cole (2014) & 1 & 7 \\
\hline Sohn et al. (2012) & 3 & 8 \\
\hline Tecchio et al. (2010) & 1 & 6 \\
\hline Waters et al. (2017) & 5 & 10 \\
\hline Williams et al. (2010) & 4 & 8 \\
\hline Abdelmoula et al. (2016) & 1 & 6 \\
\hline Kan et al. (2013) & 1 & 6 \\
\hline Oki et al. (2016) & 3 & 8 \\
\hline Radel et al. (2017) & 4 & 8 \\
\hline Williams et al. (2013) & 4 & 9 \\
\hline Frazer et al. (2016) & 3 & 8 \\
\hline Frazer et al. (2017) & 3 & 8 \\
\hline Hendy and Kidgell (2013) & 4 & 8 \\
\hline Hendy and Kidgell (2014) & 3 & 8 \\
\hline Hendy et al. (2015) & 3 & 8 \\
\hline
\end{tabular}

Higher scores represent higher quality.

postulated (Cogiamanian et al., 2007). Increases in motor cortex excitability with tDCS (Nitsche and Paulus, 2000, 2001) were not seen in sustained contractions of $20 \%$ (Cogiamanian et al., 2007; Williams et al., 2013) and 35\% (Abdelmoula et al., 2016) of maximal isometric voluntary contraction (MIVC). However, one of these studies (Williams et al., 2013) did find significant increases in MEPs during a slight contraction following tDCS suggestive of increased cortical excitability. Furthermore, Krishnan et al. (2014) demonstrated increase in EMG magnitude during elbow flexion in higher force levels at 37.5 and $50 \%$ of maximum, but not in lower levels. Improvements in force were additionally associated with increased cortical excitability as seen in studies with (Hendy and Kidgell, 2013, 2014; Hendy et al., 2015; Frazer et al., 2017) or without (Frazer et al., 2016) strength training and with (Hendy and Kidgell, 2013; Hendy et al., 2015; Frazer et al., 2016) or without (Hendy and Kidgell, 2014; Frazer et al., 2017) repeated stimulation. These studies also indicate an increase in cross-activation and decrease in short-interval intracortical inhibition as contributory factors. Conversely, other studies have failed to demonstrate MIVC improvement theorized to be due to ceiling effects of maximal muscle contractility (Kan et al., 2013) but also membrane excitability (Williams et al., 2013) as suggested by a lack of difference in MEPs (Lampropoulou and Nowicky, 2013) during elbow flexion.

\section{Safety Considerations}

Given the promising findings in improving upper limb motor performance discussed above, it is important to evaluate the safety aspects neurostimulation technology. Several literature reviews suggest tDCS is safe (Brunoni et al., 2011, 2012; Bikson et al., 2016; Fregni et al., 2016; Woods et al., 2016; Matsumoto and Ugawa, 2017). In an extensive review of tDCS safety (Bikson et al., 2016), no serious adverse events or irreversible injuries were documented in 33,200 sessions in 1,000 subjects including certain potentially vulnerable populations. Common minor side effects include "tingling" and "itching," which are typically transient and subside following stimulation, and redness, which tends to disappear after 1-2 h. For cumulative exposure, a systematic review (Nikolin et al., 2018) concluded no additional risks to subjects with repeated sessions of tDCS. Healthy subjects have received up to 30 sessions of tDCS without any serious adverse events (Paneri et al., 2015) and some neuropsychiatric patients have received over 100 sessions without any serious adverse events (Andrade, 2013). tDCS has also been shown to be safe in children with over 2,800 sessions on nearly 500 subjects showing no serious adverse effects (Bikson et al., 2016). Two additional reviews also supported these findings with no serious adverse effects observed with tDCS in children (Krishnan et al., 2015; Palm et al., 2016). On a cellular level, Nitsche et al. (2003a) examined neuron specific enolase, a protein associated with neuronal death, in subjects undergoing tDCS and revealed no change in enolase concentration following treatment. In cortical imaging studies, MRI was used to examine subjects for brain oedema, disturbance of the blood-brain barrier and structural alterations of the brain following tDCS and demonstrated no such concerns in any of their subjects (Nitsche et al., 2004). Similarly, Tadini et al. (2011) have confirmed no significant abnormal effects of tDCS on EEG. Furthermore, tDCS is recognised by the National Institute for Health and Care Excellence (NICE) as a safe option in the treatment of depression in adults. It is important to note that this safety profile is assumed only for experiments within certain stimulation protocol limits (e.g., stimulation current up to $2 \mathrm{~mA}$ ). Although these parameters are being extended (e.g., current up to $4 \mathrm{~mA}$ ) in ongoing research (Chhatbar et al., 2017), further work is required to ascertain the exact protocol limits for physiological safety. 


\section{CONCLUSIONS}

The current meta-analysis suggests that tDCS confers immediate performance benefits in dexterity tasks and exercise tasks. Importantly, these results must be interpreted with caution owing to the widespread methodological differences in the experimental domain of tDCS highlighted within this review. Whilst it is appropriate to vary methodology according to the proposed scientific question of the study and also to better appraise the physiological mechanisms of tDCS, the sheer range of methodologies currently utilised has rendered it challenging to group studies for meta-analysis. Additional research is required to delineate neural mechanisms contributing to the effect of tDCS on motor performance which will further our understanding of individual, task and study variability. As the field progresses, narrower stimulation protocols and approaching future work with an emerging standardized manner (Buch et al., 2017) will help to derive more reliable conclusions.

\section{LIMITATIONS}

The main limitation of this review lies in the considerable methodological heterogeneity of stimulation protocols, task type and reporting of outcomes. Antal and colleagues (Antal et al., 2015) accurately highlight significant limitations of metaanalysis within the field, some of which are unavoidable due to methodological variability. Accordingly, studies were restricted to those which reported data for the same outcome variable at the same post-stimulation time-point; long-term/retention effects were not within the remit of this study. Similarly, although initial

\section{REFERENCES}

Abdelmoula, A., Baudry, S., and Duchateau, J. (2016). Anodal transcranial direct current stimulation enhances time to task failure of a submaximal contraction of elbow flexors without changing corticospinal excitability. Neuroscience 322, 94-103. doi: 10.1016/j.neuroscience.2016.02.025

Ambrus, G. G., Chaieb, L., Stilling, R., Rothkegel, H., Antal, A., and Paulus, W. (2016). Monitoring transcranial direct current stimulation induced changes in cortical excitability during the serial reaction time task. Neurosci. Lett. 616, 98-104. doi: 10.1016/j.neulet.2016.01.039

Andrade, C. (2013). Once- to twice-daily, 3-year domiciliary maintenance transcranial direct current stimulation for severe, disabling, clozapinerefractory continuous auditory hallucinations in schizophrenia. J. ECT 29, 239-242. doi: 10.1097/YCT.0b013e3182843866

Angius, L., Hopker, J., and Mauger, A. R. (2017). The ergogenic effects of transcranial direct current stimulation on exercise performance. Front. Physiol. 8:90. doi: 10.3389/fphys.2017.00090

Antal, A., Keeser, D., Priori, A., Padberg, F., and Nitsche, M. A. (2015). Conceptual and procedural shortcomings of the systematic review "Evidence That Transcranial Direct Current Stimulation (tDCS) generates little-to-no reliable neurophysiologic effect beyond MEP amplitude modulation in healthy human subjects: a systematic review." Brain Stimul. 8, 846-849. doi: 10.1016/j.brs.2015. 05.010

Apšvalka, D., Ramsey, R., and Cross, E. S. (2018). Anodal tDCS over primary motor cortex provides no advantage to learning motor sequences via observation. Neural Plast. 2018:1237962. doi: 10.1155/2018/1237962

Arias, P., Corral-Bergantiños, Y., Robles-García, V., Madrid, A., Oliviero, A., and Cudeiro, J. (2016). Bilateral tDCS on primary motor cortex: effects on fast arm reaching tasks. PLoS ONE 11:e0160063. doi: 10.1371/journal.pone.0160063 analysis included all protocols to provide an overview of the effect of tDCS, further subgroup analyses of anodal motor stimulation was performed to draw more precise conclusions. Further restricting studies to the same montage, current density and duration would limit available data to an extent that statistical analysis would not be possible or appropriate. Although the present analysis combined single- and multi-session experiments, we deemed this to represent the overall impact of tDCS and where possible, data was extracted after the first session only. Although different tasks were combined for RT and ET analyses, this approach is similar to other published tDCS-related meta-analysis (Dedoncker et al., 2016) and a random-effects model analysis was performed to account for heterogeneity. Finally, individual studies included in the meta-analyses had a small sample size which could potentially reduce the power of analysis.

\section{AUTHOR CONTRIBUTIONS}

RP, HS, HA, and DL designed the structure and scope of the review. RP, JA, and AP collected review articles. RP prepared the manuscript draft. All authors reviewed and revised the manuscript.

\section{FUNDING}

This research was funded by the NIHR Imperial Biomedical Research Centre (BRC; grant 1215-20013). The views expressed are those of the authors and not necessarily those of the NIHR of the Department of Health and Social Care.

Baker, J. M., Rorden, C., and Fridriksson, J. (2010). Using transcranial directcurrent stimulation to treat stroke patients with Aphasia. Stroke 41, 1229-1236. doi: 10.1161/STROKEAHA.109.576785

Bandeira, I. D., Guimarães, R. S. Q., Jagersbacher, J. G., Barretto, T. L., de JesusSilva, J. R., Santos, S. N., et al. (2016). Transcranial direct current stimulation in children and adolescents with attention-deficit/hyperactivity disorder (ADHD). J. Child Neurol. 31, 918-924. doi: 10.1177/0883073816630083

Bastani, A., and Jaberzadeh, S. (2012). Does anodal transcranial direct current stimulation enhance excitability of the motor cortex and motor function in healthy individuals and subjects with stroke: a systematic review and metaanalysis. Clin. Neurophysiol. 123, 644-657. doi: 10.1016/j.clinph.2011.08.029

Bikson, M., Grossman, P., Thomas, C., Zannou, A. L., Jiang, J., Adnan, T., et al. (2016). Safety of transcranial direct current stimulation: evidence based update 2016. Brain Stimul. 9, 641-661. doi: 10.1016/j.brs.2016.06.004

Bindman, L. J., Lippold, O. C. J., and Redfearn, J. W. T. (1964). The action of brief polarizing currents on the cerebral cortex of the rat (1) during current flow and (2) in the production of long-lasting after-effects. J. Physiol. 172, 369-382. doi: 10.1113/jphysiol.1964.sp007425

Block, H., and Celnik, P. (2013). Stimulating the cerebellum affects visuomotor adaptation but not intermanual transfer of learning. Cereblleum 12, 781-793. doi: 10.1007/s12311-013-0486-7

Boggio, P. S., Castro, L. O., Savagim, E. A., Braite, R., Cruz, V. C., Rocha, R. R., et al. (2006). Enhancement of non-dominant hand motor function by anodal transcranial direct current stimulation. Neurosci. Lett. 404, 232-236. doi: 10.1016/j.neulet.2006.05.051

Breitling, C., Zaehle, T., Dannhauer, M., Bonath, B., Tegelbeckers, J., Flechtner, H. H., et al. (2016). Improving interference control in ADHD patients with transcranial direct current stimulation (tDCS). Front. Cell. Neurosci. 10:72. doi: $10.3389 /$ fncel.2016.00072 
Brunoni, A. R., Amadera, J., Berbel, B., Volz, M. S., Rizzerio, B. G., and Fregni, F. (2011). A systematic review on reporting and assessment of adverse effects associated with transcranial direct current stimulation. Int. J. Neuropsychopharmacol. 14, 1133-1145. doi: 10.1017/S1461145710001690

Brunoni, A. R., Nitsche, M. A., Bolognini, N., Bikson, M., Wagner, T., Merabet, L., et al. (2012). Clinical research with transcranial direct current stimulation (tDCS): challenges and future directions. Brain Stimul. 5, 175-195. doi: 10.1016/j.brs.2011.03.002

Buch, E. R., Santarnecchi, E., Antal, A., Born, J., Celnik, P. A., Classen, J., et al. (2017). Effects of tDCS on motor learning and memory formation: a consensus and critical position paper. Clin. Neurophysiol. 128, 589-603. doi: 10.1016/j.clinph.2017.01.004

Cantarero, G., Spampinato, D., Reis, J., Ajagbe, L., Thompson, T., Kulkarni, K., et al. (2015). Cerebellar direct current stimulation enhances on-line motor skill acquisition through an effect on accuracy. J. Neurosci. 35, 3285-3290. doi: 10.1523/JNEUROSCI.2885-14.2015

Carlsen, A. N., Eagles, J. S., and MacKinnon, C. D. (2015). Transcranial direct current stimulation over the supplementary motor area modulates the preparatory activation level in the human motor system. Behav. Brain Res. 279, 68-75. doi: 10.1016/j.bbr.2014.11.009

Carter, M. J., Maslovat, D., and Carlsen, A. N. (2017). Intentional switches between coordination patterns are faster following anodal-tDCS applied over the supplementary motor area. Brain Stimul. 10, 162-164. doi: 10.1016/j.brs.2016.11.002

Chhatbar, P. Y., Chen, R., Deardorff, R., Dellenbach, B., Kautz, S. A., George, M. S., et al. (2017). Safety and tolerability of transcranial direct current stimulation to stroke patients - A phase I current escalation study. Brain Stimul. 10, 553-559. doi: 10.1016/j.brs.2017.02.007

Chothia, M., Doeltgen, S., and Bradnam, L. V. (2016). Anodal cerebellar direct current stimulation reduces facilitation of propriospinal neurons in healthy humans. Brain Stimul. 9, 364-371. doi: 10.1016/j.brs.2016.01.002

Ciechanski, P., Cheng, A., Lopushinsky, S., Hecker, K., Gan, L. S., Lang, S., et al. (2017). Effects of transcranial direct-current stimulation on neurosurgical skill acquisition: a randomized controlled trial. World Neurosurg. 108, 876-884.e4. doi: 10.1016/j.wneu.2017.08.123

Cogiamanian, F., Marceglia, S., Ardolino, G., Barbieri, S., and Priori, A. (2007). Improved isometric force endurance after transcranial direct current stimulation over the human motor cortical areas. Eur. J. Neurosci. 26, 242-249. doi: 10.1111/j.1460-9568.2007.05633.x

Convento, S., Bolognini, N., Fusaro, M., Lollo, F., and Vallar, G. (2014). Neuromodulation of parietal and motor activity affects motor planning and execution. CORTEX 57, 51-59. doi: 10.1016/j.cortex.2014.03.006

Cuypers, K., Leenus, D. J. F., van den Berg, F. E., Nitsche, M. A., Thijs, H., Wenderoth, N., et al. (2013). Is motor learning mediated by tDCS intensity? PLoS ONE 8:e67344. doi: 10.1371/journal.pone.0067344

Dedoncker, J., Brunoni, A. R., Baeken, C., and Vanderhasselt, M. A. (2016). A systematic review and meta-analysis of the effects of transcranial direct current stimulation (tDCS) over the dorsolateral prefrontal cortex in healthy and neuropsychiatric samples: influence of stimulation parameters. Brain Stimul. 9, 501-517. doi: 10.1016/j.brs.2016.04.006

Doppelmayr, M., Pixa, N. H., and Steinberg, F. (2016). Cerebellar, but not motor or parietal, high-density anodal transcranial direct current stimulation facilitates motor adaptation. J. Int. Neuropsychol. Soc. 22, 928-936. doi: $10.1017 /$ S1355617716000345

Doyon, J., Bellec, P., Amsel, R., Penhune, V., Monchi, O., Carrier, J., et al. (2009). Contributions of the basal ganglia and functionally related brain structures to motor learning. Behav. Brain Res. 199, 61-75. doi: 10.1016/j.bbr.2008.11.012

Doyon, J., Song, A. W., Karni, A., Lalonde, F., Adams, M. M., and Ungerleider, L. G. (2002). Experience-dependent changes in cerebellar contributions to motor sequence learning. Proc. Natl. Acad. Sci. U.S.A. 99, 1017-1022. doi: 10.1073/pnas.022615199

Dumel, G., Bourassa, M.-È., Charlebois-Plante, C., Desjardins, M., Doyon, J., Saint-Amour, D., et al. (2018). Multisession anodal transcranial direct current stimulation induces motor cortex plasticity enhancement and motor learning generalization in an aging population. Clin. Neurophysiol. 129, 494-502. doi: 10.1016/j.clinph.2017.10.041

Dumel, G., Bourassa, M. E., Desjardins, M., Voarino, N., Charlebois-Plante, C., Doyon, J., et al. (2016). Multisession anodal tDCS protocol improves motor system function in an aging population. Neural Plast. 2016, 15-18. doi: 10.1155/2016/5961362

Edwards, D. J., Cortes, M., Wortman-Jutt, S., Putrino, D., Bikson, M., Thickbroom, G., et al. (2017). Transcranial direct current stimulation and sports performance. Front. Hum. Neurosci. 11:243. doi: 10.3389/fnhum.2017.00243

Ehsani, F., Bakhtiary, A. H., Jaberzadeh, S., Talimkhani, A., and Hajihasani, A. (2016). Differential effects of primary motor cortex and cerebellar transcranial direct current stimulation on motor learning in healthy individuals: a randomized double-blind sham-controlled study. Neurosci. Res. 112, 10-19. doi: 10.1016/j.neures.2016.06.003

Fagerlund, A. J., Hansen, O. A., and Aslaksen, P. M. (2015). Transcranial direct current stimulation as a treatment for patients with fibromyalgia. Pain 156, 62-71. doi: 10.1016/j.pain.0000000000000006

Fenton, B. W., Palmieri, P. A., Boggio, P., Fanning, J., and Fregni, F. (2009). A preliminary study of transcranial direct current stimulation for the treatment of refractory chronic pelvic pain. Brain Stimul. 2, 103-107. doi: 10.1016/j.brs.2008.09.009

Ferrucci, R., Brunoni, A. R., Parazzini, M., Vergari, M., Rossi, E., Fumagalli, M., et al. (2013). Modulating human procedural learning by cerebellar transcranial direct current stimulation centro clinico per la neurostimolazione, le neurotecnologie ed i disordini del movimento, fondazione IRCCS Ca' granda. Cerebellum 12, 485-492. doi: 10.1007/s12311-012-0436-9

Floyer-Lea, A., and Matthews, P. M. (2005). Distinguishable brain activation networks for short- and long-term motor skill learning. J. Neurophysiol. 94, 512-518. doi: 10.1152/jn.00717.2004

Focke, J., Kemmet, S., Krause, V., Keitel, A., and Pollok, B. (2017). Cathodal transcranial direct current stimulation (tDCS) applied to the left premotor cortex (PMC) stabilizes a newly learned motor sequence. Behav. Brain Res. 316, 87-93. doi: 10.1016/j.bbr.2016.08.032

Frazer, A., Williams, J., Spittles, M., Rantalainen, T., and Kidgell, D. (2016). Anodal transcranial direct current stimulation of the motor cortex increases cortical voluntary activation and neural plasticity. Muscle Nerve 54, 903-913. doi: $10.1002 /$ mus. 25143

Frazer, A. K., Williams, J., Spittle, M., and Kidgell, D. J. (2017). Cross-education of muscular strength is facilitated by homeostatic plasticity. Eur. J. Appl. Physiol. 117, 665-677. doi: 10.1007/s00421-017-3538-8

Fregni, F., Boggio, P. S., Lima, M. C., Ferreira, M. J. L., Wagner, T., Rigonatti, S. P., et al. (2006). A sham-controlled, phase II trial of transcranial direct current stimulation for the treatment of central pain in traumatic spinal cord injury. Pain 122, 197-209. doi: 10.1016/j.pain.2006.02.023

Fregni, F., Nitsche, M., Loo, C., Brunoni, A., Marangolo, P., Leite, J., et al. (2016). Regulatory considerations for the clinical and research use of transcranial direct current stimulation (tDCS): review and recommendations from an expert panel. Clin. Res. Regul. Aff. 32, 22-35. doi: 10.3109/10601333.2015.9 80944

Furuya, S., Klaus, M., Nitsche, M. A., Paulus, W., and Altenmüller, E. (2014). Ceiling effects prevent further improvement of transcranial stimulation in skilled musicians. J. Neurosci. 34, 13834-13839. doi: 10.1523/JNEUROSCI.1170-14.2014

Galea, J. M., Vazquez, A., Pasricha, N., de Xivry, J.-J. O., and Celnik, P. (2011) Dissociating the roles of the cerebellum and motor cortex during adaptive learning: the motor cortex retains what the cerebellum learns. Cereb. Cortex 21, 1761-1770. doi: 10.1093/cercor/bhq246

Giordano, J., Bikson, M., Kappenman, E. S., Clark, V. P., Branch Coslett, H., Hamblin, M. R., et al. (2017). Mechanisms and effects of transcranial direct current stimulation. Dose-Response 15:1559325816685467. doi: $10.1177 / 1559325816685467$

Gomes-Osman, J., and Field-Fote, E. C. (2013). Bihemispheric anodal corticomotor stimulation using transcranial direct current stimulation improves bimanual typing task performance. J. Mot. Behav. 45, 361-367. doi: 10.1080/00222895.2013.808604

Goodwill, A. M., Reynolds, J., Daly, R. M., and Kidgell, D. J. (2013). Formation of cortical plasticity in older adults following tDCS and motor training. Front. Aging Neurosci. 5:87. doi: 10.3389/fnagi.2013. 00087

Hardwick, R. M., and Celnik, P. A. (2014). Cerebellar direct current stimulation enhances motor learning in older adults. Neurobiol. Aging 35, 2217-2221. doi: 10.1016/j.neurobiolaging.2014.03.030 
Hardwick, R. M., Rottschy, C., Miall, R. C., and Eickhoff, S. B. (2013). A quantitative meta-analysis and review of motor learning in the human brain. Neuroimage 67, 283-297. doi: 10.1016/j.neuroimage.2012.11.020

Hashemirad, F., Fitzgerald, P. B., Zoghi, M., and Jaberzadeh, S. (2017). Singlesession anodal tDCS with small-size stimulating electrodes over frontoparietal superficial sites does not affect motor sequence learning. Front. Hum. Neurosci. 11:153. doi: 10.3389/fnhum.2017.00153

Hashemirad, F., Zoghi, M., Fitzgerald, P. B., and Jaberzadeh, S. (2016). The effect of anodal transcranial direct current stimulation on motor sequence learning in healthy individuals: a systematic review and meta-analysis. Brain Cogn. 102, 1-12. doi: 10.1016/j.bandc.2015.11.005

Heise, K.-F., Niehoff, M., Feldheim, J.-F., Liuzzi, G., Gerloff, C., and Hummel, F. C. (2014). Differential behavioral and physiological effects of anodal transcranial direct current stimulation in healthy adults of younger and older age. Front. Aging Neurosci. 6:146. doi: 10.3389/fnagi.2014.00146

Hendy, A. M., and Kidgell, D. J. (2013). Anodal tDCS applied during strength training enhances motor cortical plasticity. Med. Sci. Sport. Exerc. 45, 1721-1729. doi: 10.1249/MSS.0b013e31828d2923

Hendy, A. M., and Kidgell, D. J. (2014). Anodal-tDCS applied during unilateral strength training increases strength and corticospinal excitability in the untrained homologous muscle. Exp. Brain Res. 232:3243. doi: $10.1007 / \mathrm{s} 00221-014-4016-8$

Hendy, A. M., Teo, W., and Kidgell, D. J. (2015). Anodal transcranial direct current stimulation prolongs the cross-education of strength and corticomotor plasticity. Med. Sci. Sport. Exerc. 47, 1788-1797. doi: 10.1249/MSS.0000000000000600

Herzfeld, D. J., Pastor, D., Haith, A. M., Rossetti, Y., Shadmehr, R., and O'Shea, J. (2014). Contributions of the cerebellum and the motor cortex to acquisition and retention of motor memories. Neuroimage 98, 147-158. doi: 10.1016/j.neuroimage.2014.04.076

Higgins, J. P. T., and Green, S. (eds). (2011). Cochrane Handbook for Systematic Reviews of Interventions Version 5.1.0 [updated March 2011]. The Cochrane Collaboration. Available online at: www.handbook.cochrane.org

Horvath, J. C., Carter, O., and Forte, J. D. (2016). No significant effect of transcranial direct current stimulation (tDCS) found on simple motor reaction time comparing 15 different simulation protocols. Neuropsychologia 91, 544-552. doi: 10.1016/j.neuropsychologia.2016.09.017

Huang, L., Deng, Y., Zheng, X., and Liu, Y. (2019). Transcranial direct current stimulation with halo sport enhances repeated sprint cycling and cognitive performance. Front. Physiol. 10:118. doi: 10.3389/fphys.2019.00118

Hummel, F. C., Heise, K., Celnik, P., Floel, A., Gerloff, C., and Cohen, L. G. (2010). Facilitating skilled right hand motor function in older subjects by anodal polarization over the left primary motor cortex. Neurobiol. Aging 31, 2160-2168. doi: 10.1016/j.neurobiolaging.2008.12.008

Jadad, A., Moore, R., Carroll, D., Jenkinson, C., Reynolds, J., Gavaghan, D., et al. (1996). Assessing the quality of reports of randomized clinical trials: is blinding necessary? Control. Clin. Trials 17, 1-12. doi: 10.1016/0197-2456(95)00134-4

Jenkins, I. H., Brooks, D. J., Nixon, P. D., Frackowiak, R. S. J., and Passingham, F. E. (1994). Motor sequence learning: a study with positron emission tomography. J. Neurosci. 14, 3775-3790. doi: 10.1523/JNEUROSCI.14-06-03775.1994

Kaminski, E., Steele, C. J., Hoff, M., Gundlach, C., Rjosk, V., Sehm, B., et al. (2016). Transcranial direct current stimulation (tDCS) over primary motor cortex leg area promotes dynamic balance task performance. Clin. Neurophysiol. 127, 2455-2462. doi: 10.1016/j.clinph.2016.03.018

Kan, B., Dundas, J. E., and Nosaka, K. (2013). Effect of transcranial direct current stimulation on elbow flexor maximal voluntary isometric strength and endurance. Appl. Physiol. Nutr. Metab. 38, 734-739. doi: 10.1139/apnm-2012-0412

Kang, E. K., and Paik, N.-J. (2011). Effect of a tDCS electrode montage on implicit motor sequence learning in healthy subjects. Exp. Transl. Stroke Med. 3:4. doi: 10.1186/2040-7378-3-4

Kantak, S. S., Mummidisetty, C. K., and Stinear, J. W. (2012). Primary motor and premotor cortex in implicit sequence learning - evidence for competition between implicit and explicit human motor memory systems. Eur. J. Neurosci. 36, 2710-2715. doi: 10.1111/j.1460-9568.2012.08175.x

Karni, A., Meyer, G., Jezzard, P., Adams, M. M., Turner, R., and Ungerleider, L. G. (1995). Functional MRI evidence for adult motor cortex plasticity during motor skill learning. Nature 377, 155-158. doi: 10.1038/377155a0
Karok, S., Fletcher, D., and Witney, A. G. (2017). Task-specificity of unilateral anodal and dual-M1 tDCS effects on motor learning. Neuropsychologia 94, 84-95. doi: 10.1016/j.neuropsychologia.2016.12.002

Karok, S., and Witney, A. G. (2013). Enhanced motor learning following taskconcurrent dual transcranial direct current stimulation. PLOS ONE 8:e85693. doi: 10.1371/journal.pone.0085693

Kaski, D., Dominguez, R., Allum, J., Islam, A., and Bronstein, A. (2014). Combining physical training with transcranial direct current stimulation to improve gait in Parkinson's disease: a pilot randomized controlled study. Clin. Rehabil. 28, 1115-1124. doi: 10.1177/0269215514534277

Kidgell, D. J., Goodwill, A. M., Frazer, A. K., and Daly, R. M. (2013). Induction of cortical plasticity and improved motor performance following unilateral and bilateral transcranial direct current stimulation of the primary motor cortex. BMC Neurosci. 14:64. doi: 10.1186/1471-2202-14-64

Koyama, S., Tanaka, S., Tanabe, S., and Sadato, N. (2015). Dual-hemisphere transcranial direct current stimulation over primary motor cortex enhances consolidation of a ballistic thumb movement. Neurosci. Lett. 588, 49-53. doi: 10.1016/j.neulet.2014.11.043

Krishnan, C., Ranganathan, R., Kantak, S. S., Dhaher, Y. Y., and Rymer, W. Z. (2014). Anodal transcranial direct current stimulation alters elbow flexor muscle recruitment strategies. Brain Stimul. 7, 443-450. doi: 10.1016/j.brs.2014.01.057

Krishnan, C., Santos, L., Peterson, M. D., and Ehinger, M. (2015). Safety of noninvasive brain stimulation in children and adolescents. Brain Stimul. 8, 76-87. doi: 10.1016/j.brs.2014.10.012

Lampropoulou, S. I., and Nowicky, A. V. (2013). The effect of transcranial direct current stimulation on perception of effort in an isolated isometric elbow flexion task. Motor Control 17, 412-426. doi: 10.1123/mcj.17.4.412

Lang, N., Siebner, H. R., Ward, N. S., Lee, L., Nitsche, M. A., Paulus, W., et al. (2005). How does transcranial DC stimulation of the primary motor cortex alter regional neuronal activity in the human brain? Europe PMC funders group. Eur. J. Neurosci. 22, 495-504. doi: 10.1111/j.1460-9568.2005.04233.x

Lattari, E., Oliveira, B. R. R., Monteiro Júnior, R. S., Marques Neto, S. R., Oliveira, A. J., Maranhão Neto, G. A., et al. (2018). Acute effects of single dose transcranial direct current stimulation on muscle strength: a systematic review and meta-analysis. PLoS ONE 13:e0209513. doi: 10.1371/journal.pone.0209513

Leite, J., Carvalho, S., Fregni, F., and Gonçalves, Ó. F. (2011). Taskspecific effects of tDCS-induced cortical excitability changes on cognitive and motor sequence set shifting performance. PLoS ONE 6:e24140. doi: 10.1371/journal.pone. 0024140

Liebetanz, D., Nitsche, M. A., Tergau, F., and Paulus, W. (2002). Pharmacological approach to the mechanisms of transcranial DC-stimulation-induced after-effects of human motor cortex excitability. Brain 125, 2238-2247. doi: 10.1093/brain/awf238

Lindenberg, R., Nachtigall, L., Meinzer, M., Sieg, M. M., and Flöel, A. (2013). Differential effects of dual and unihemispheric motor cortex stimulation in older adults. J. Neurosci. 33, 9176-9183. doi: 10.1523/JNEUROSCI.0055-13.2013

Lindenberg, R., Sieg, M. M., Meinzer, M., Nachtigall, L., and Flöel, A. (2016). Neural correlates of unihemispheric and bihemispheric motor cortex stimulation in healthy young adults. Neuroimage 140, 141-149. doi: 10.1016/j.neuroimage.2016.01.057

Loo, C. K., Alonzo, A., Martin, D., Mitchell, P. B., Galvez, V., and Sachdev, P. (2012). Transcranial direct current stimulation for depression: 3-week, randomised, sham-controlled trial. Br. J. Psychiatry 200, 52-59. doi: 10.1192/bjp.bp.111.097634

Lopez-Alonso, V., del Olmo, F. M., Liew, S.-L., Fernández del Olmo, M., and Cheeran, B. (2018). A preliminary comparison of motor learning across different non-invasive brain stimulation paradigms shows no consistent modulations. Front. Neurosci. 1:253. doi: 10.3389/fnins.2018.00253

Machado, D. G. D. S., Unal, G., Andrade, S. M., Moreira, A., and Altimari, L. R. (2019). Effect of transcranial direct current stimulation on exercise performance: a systematic review and meta-analysis. Brain Stimul. 12:593-605. doi: 10.1016/j.brs.2018.12.227

Marquez, J., Conley, A., Karayanidis, F., Lagopoulos, J., and Parsons, M. (2015). Anodal direct current stimulation in the healthy aged: effects determined by the hemisphere stimulated. Restor. Neurol. Neurosci. 33, 509-519. doi: 10.3233/RNN-140490 
Matsumoto, H., and Ugawa, Y. (2017). Adverse events of tDCS and tACS: a review. Clin. Neurophysiol. Pract. 2, 19-25. doi: 10.1016/j.cnp.2016.12.003

Matsuo, A., Maeoka, H., Hiyamizu, M., Shomoto, K., Morioka, S., and Seki, K. (2011). Enhancement of precise hand movement by transcranial direct current stimulation. Neuroreport 22, 78-82. doi: 10.1097/WNR.0b013e32834298b3

Mccambridge, A. B., Stinear, J. W., and Byblow, W. D. (2016). Neurophysiological and behavioural effects of dual-hemisphere transcranial direct current stimulation on the proximal upper limb. Exp. Brain Res. 234, 1419-1428. doi: 10.1007/s00221-015-4547-7

Medina, J., and Cason, S. (2017). No evidential value in samples of transcranial direct current stimulation (tDCS) studies of cognition and working memory in healthy populations. Cortex 94, 131-141. doi: 10.1016/j.cortex.2017.06.021

Mizuguchi, N., Katayama, T., and Kanosue, K. (2018). The effect of cerebellar transcranial direct current stimulation on a throwing task depends on individual level of task performance. Neuroscience 371:119-125. doi: 10.1016/j.neuroscience.2017.11.048

Naros, G., Geyer, M., Koch, S., Mayr, L., Ellinger, T., Grimm, F., et al. (2016). Enhanced motor learning with bilateral transcranial direct current stimulation: impact of polarity or current flow direction? Clin. Neurophysiol. 127, 2119-2126. doi: 10.1016/j.clinph.2015.12.020

Nikolin, S., Huggins, C., Martin, D., Alonzo, A., and Loo, C. K. (2018). Safety of repeated sessions of transcranial direct current stimulation: a systematic review. Brain Stimul. 11, 278-288. doi: 10.1016/j.brs.2017.10.020

Nilsson, J., Lebedev, A. V., Rydström, A., and Lövdén, M. (2017). Direct-current stimulation does little to improve the outcome of working memory training in older adults. Psychol. Sci. 28, 907-920. doi: 10.1177/0956797617698139

Nitsche, M. A., Jakoubkova, M., Thirugnanasambandam, N., Schmalfuss, L., Hullemann, S., Sonka, K., et al. (2010). Contribution of the premotor cortex to consolidation of motor sequence learning in humans during sleep. $J$. Neurophysiol. 104, 2603-2614. doi: 10.1152/jn.00611.2010

Nitsche, M. A., Niehaus, L., Hoffmann, K. T., Hengst, S., Liebetanz, D., Paulus, W., et al. (2004). MRI study of human brain exposed to weak direct current stimulation of the frontal cortex. Clin. Neurophysiol. 115, 2419-2423. doi: 10.1016/j.clinph.2004.05.001

Nitsche, M. A., Nitsche, M. S., Klein, C. C., Tergau, F., Rothwell, J. C., and Paulus, W. (2003a). Level of action of cathodal DC polarisation induced inhibition of the human motor cortex. Clin. Neurophysiol. 114, 600-604. doi: 10.1016/S1388-2457(02)00412-1

Nitsche, M. A., and Paulus, W. (2000). Excitability changes induced in the human motor cortex by weak transcranial direct current stimulation. J. Physiol. 527, 633-639. doi: 10.1111/j.1469-7793.2000.t01-1-00633.x

Nitsche, M. A., and Paulus, W. (2001). Sustained excitability elevations induced by transcranial DC motor cortex stimulation in humans. Neurology 57, 1899-1901. doi: 10.1212/WNL.57.10.1899

Nitsche, M. A., Schauenburg, A., Lang, N., Liebetanz, D., Exner, C., Paulus, W., et al. (2003b). Facilitation of implicit motor learning by weak transcranial direct current stimulation of the primary motor cortex in the human facilitation of implicit motor learning by weak transcranial direct current stimulation of the primary motor cortex in the human. J. Cogn. Neurosci. 15, 619-626. doi: $10.1162 / 089892903321662994$

Okano, A. H., Fontes, E. B., Montenegro, R. A., Farinatti, P., de, T. V., Cyrino, E. S., et al. (2015). Brain stimulation modulates the autonomic nervous system, rating of perceived exertion and performance during maximal exercise. $\mathrm{Br}$. J. Sports Med. 49, 1213-1218. doi: 10.1136/bjsports-2012-091658

Oki, K., Mahato, N. K., Nakazawa, M., Amano, S., France, C. R., Russ, D. W., et al. (2016). Preliminary evidence that excitatory transcranial direct current stimulation extends time to task failure of a sustained, submaximal muscular contraction in older adults. J. Gerontol. Biol. Sci. Med. Sci. 71, 1109-1112. doi: 10.1093/gerona/glw011

Palm, U., Schiller, C., Fintescu, Z., Obermeier, M., Keeser, D., Reisinger, E., et al. (2012). Transcranial direct current stimulation in treatment resistant depression: a randomized double-blind, placebo-controlled study. Brain Stimul. 5, 242-251. doi: 10.1016/j.brs.2011.08.005

Palm, U., Segmiller, F. M., Epple, A. N., Freisleder, F.-J., Koutsouleris, N., SchulteKörne, G., et al. (2016). Transcranial direct current stimulation in children and adolescents: a comprehensive review. J. Neural Transm. 123, 1219-1234. doi: 10.1007/s00702-016-1572-z
Paneri, B., Khadka, N., Patel, V., Thomas, C., Tyler, W., Parra, L. C., et al. (2015). The tolerability of transcranial electrical stimulation used across extended periods in a naturalistic context by healthy individuals. PeerJ. 3:e1097v2. doi: $10.7287 /$ peerj.preprints.1097v2

Panouilieres, M. T. N., Joundi, R. A., Brittain, J.-S., and Jenkinson, N. (2015). The journal of physiology neuroscience C 2015 the authors. J. Physiol. 593, 3645-3655. doi: 10.1113/JP270484

Parikh, P. J., and Cole, K. J. (2014). Effects of transcranial direct current stimulation in combination with motor practice on dexterous grasping and manipulation in healthy older adults. Physiol. Rep. 2:e00255. doi: 10.1002/phy2.255

Park, S.-B., Sung, D. J., Kim, B., Kim, S., and Han, J.-K. (2019). Transcranial direct current Stimulation of motor cortex enhances running performance. PLOS ONE 14:e0211902. doi: 10.1371/journal.pone.0211902

Pixa, N. H., and Pollok, B. (2018). Effects of tDCS on bimanual motor skills: a brief review. Front. Behav. Neurosci. 12:63. doi: 10.3389/fnbeh.2018.00063

Pixa, N. H., Steinberg, F., and Doppelmayr, M. (2017a). Effects of high-definition anodal transcranial direct current stimulation applied simultaneously to both primary motor cortices on bimanual sensorimotor performance. Front. Behav. Neurosci. 11:130. doi: 10.3389/fnbeh.2017.00130

Pixa, N. H., Steinberg, F., and Doppelmayr, M. (2017b). High-definition transcranial direct current stimulation to both primary motor cortices improves unimanual and bimanual dexterity. Neurosci. Lett. 643, 84-88. doi: 10.1016/j.neulet.2017.02.033

Prichard, G., Weiller, C., Fritsch, B., and Reis, J. (2014). Effects of different electrical brain stimulation protocols on subcomponents of motor skill learning. Brain Stimul. 7, 532-540. doi: 10.1016/j.brs.2014.04.005

Radel, R., Tempest, G., Denis, G., Besson, P., and Zory, R. (2017). Extending the limits of force endurance: stimulation of the motor or the frontal cortex? Cortex 97, 96-108. doi: 10.1016/j.cortex.2017.09.026

Reis, J., and Fritsch, B. (2011). Modulation of motor performance and motor learning by transcranial direct current stimulation. Curr. Opin. Neurol. 24, 590-596. doi: 10.1097/WCO.0b013e32834c3db0

Reis, J., Schambra, H. M., Cohen, L. G., Buch, E. R., Fritsch, B., Zarahn, E., et al. (2009). Noninvasive cortical stimulation enhances motor skill acquisition over multiple days through an effect on consolidation. Proc. Natl. Acad. Sci. U.S.A. 106, 1590-1595. doi: 10.1073/pnas.0805413106

Rroji, O., van Kuyck, K., Nuttin, B., and Wenderoth, N. (2015). Anodal tDCS over the primary motor cortex facilitates long-term memory formation reflecting use-dependent plasticity. PLOS ONE 10:e0127270. doi: 10.1371/journal.pone.0127270

Rumpf, J.-J., Wegscheider, M., Hinselmann, K., Fricke, C., King, B. R., Weise, D., et al. (2017). Enhancement of motor consolidation by post-training transcranial direct current stimulation in older people. Neurobiol. Aging 49, 1-8. doi: 10.1016/j.neurobiolaging.2016.09.003

Samaei, A., Ehsani, F., Zoghi, M., Hafez Yosephi, M., and Jaberzadeh, S. (2017). Online and offline effects of cerebellar transcranial direct current stimulation on motor learning in healthy older adults: a randomized double-blind shamcontrolled study. Eur. J. Neurosci. 45, 1177-1185. doi: 10.1111/ejn.13559

Saruco, E., Di Rienzo, F., Nunez-Nagy, S., Rubio-Gonzalez, M. A., Jackson, P. L., Collet, C., et al. (2017). Anodal tDCS over the primary motor cortex improves motor imagery benefits on postural control: a pilot study. Sci. Rep. 7:480. doi: 10.1038/s41598-017-00509-w

Saucedo Marquez, C. M., Zhang, X., Swinnen, S. P., Meesen, R., and Wenderoth, N. (2013). Task-specific effect of transcranial direct current stimulation on motor learning. Front. Hum. Neurosci. 7:333. doi: 10.3389/fnhum.2013.00333

Schambra, H. M., Abe, M., Luckenbaugh, D. A., Reis, J., Krakauer, J. W., and Cohen, L. G. (2011). Probing for hemispheric specialization for motor skill learning: a transcranial direct current stimulation study. J. Neurophysiol. 106, 652-661. doi: 10.1152/jn.00210.2011

Schmidt, S., Fleischmann, R., Bathe-Peters, R., Irlbacher, K., and Brandt, S. A. (2013). Evolution of premotor cortical excitability after cathodal inhibition of the primary motor cortex: a sham-controlled serial navigated TMS study. PLoS ONE 8:e57425. doi: 10.1371/journal.pone.0057425

Shimizu, R. E., Wu, A. D., Samra, J. K., and Knowlton, B. J. (2017). The impact of cerebellar transcranial direct current stimulation (tDCS) on learning finemotor sequences. Philos. Trans. R. Soc. Lond. Ser. B Biol. Sci. 372:20160050. doi: $10.1098 /$ rstb.2016.0050 
Simonsmeier, B. A., Grabner, R. H., Hein, J., Krenz, U., and Schneider, M. (2018). Electrical brain stimulation (tES) improves learning more than performance: a meta-analysis. Neurosci. Biobehav. Rev. 84, 171-181. doi: 10.1016/j.neubiorev.2017.11.001

Sohn, M. K., Kim, B. O., and Song, H. T. (2012). Effect of stimulation polarity of transcranial direct current stimulation on non-dominant hand function. Ann. Rehabil. Med. 36, 1-7. doi: 10.5535/arm.2012.36.1.1

Stagg, C. J., Jayaram, G., Pastor, D., Kincses, Z. T., Matthews, P. M., and JohansenBerg, H. (2011). Polarity and timing-dependent effects of transcranial direct current stimulation in explicit motor learning. Neuropsychologia 49, 800-804. doi: 10.1016/j.neuropsychologia.2011.02.009

Summers, R. L. S., Chen, M., Hatch, A., Kimberley, T. J., Perrey, S., Brighina, F., et al. (2018). Cerebellar transcranial direct current stimulation modulates corticospinal excitability during motor training. Front. Hum. Neurosci. 12:118. doi: 10.3389/fnhum.2018.00118

Tadini, L., El-Nazer, R., Brunoni, A. R., Williams, J., Carvas, M., Boggio, P., et al. (2011). Cognitive, mood, and electroencephalographic effects of noninvasive cortical stimulation with weak electrical currents. J. ECT 27, 134-140. doi: 10.1097/YCT.0b013e3181e631a8

Tan, A., Ashrafian, H., Scott, A. J., Mason, S. E., Harling, L., Athanasiou, T., et al. (2016). Robotic surgery: disruptive innovation or unfulfilled promise? A systematic review and meta-analysis of the first 30 years. Surg. Endosc. 30, 4330-52. doi: 10.1007/s00464-016-4752-x

Taubert, M., Stein, T., Kreutzberg, T., Stockinger, C., Hecker, L., Focke, A., et al. (2016). Remote effects of non-invasive cerebellar stimulation on error processing in motor re-learning. Brain Stimul. 9, 692-699. doi: 10.1016/j.brs.2016.04.007

Tecchio, F., Zappasodi, F., Assenza, G., Tombini, M., Vollaro, S., Barbati, G., et al. (2010). Anodal transcranial direct current stimulation enhances procedural consolidation. J. Neurophysiol. 104, 1134-1140. doi: 10.1152/jn.00661.2009

Toni, I., Krams, M., Turner, R., and Passingham, R. (1998). The time course of changes during motor sequence learning: a whole-brain fMRI study. Neuroimage 8, 50-61. doi: 10.1006/nimg.1998.0349

Ungerleider, L. G., Doyon, J., and Karni, A. (2002). Imaging brain plasticity during motor skill learning. Neurobiol. Learn. Mem. 78, 553-564. doi: 10.1006/nlme.2002.4091

van Tulder, M., Furlan, A., Bombardier, C., and Bouter, L. (2003) Editorial Board of the Cochrane Collaboration Back Review G. Updated Method Guidelines for Systematic Reviews in the Cochrane Collaboration Back Review Group. Spine 28, 1290-1299. doi: 10.1097/01.BRS.0000065484.95996.AF

Vergallito, A., Romero Lauro, L. J., Bonandrini, R., Zapparoli, L., Danelli, L., and Berlingeri, M. (2018). What is difficult for you can be easy for me. Effects of increasing individual task demand on prefrontal lateralization: a tDCS study. Neuropsychologia 109, 283-294. doi: 10.1016/j.neuropsychologia.2017.12.038

Vines, B. W., Cerruti, C., and Schlaug, G. (2008a). Dual-hemisphere tDCS facilitates greater improvements for healthy subjects' non-dominant hand compared to uni-hemisphere stimulation. BMC Neurosci. 9:103. doi: 10.1186/1471-2202-9-103

Vines, B. W., Nair, D., and Schlaug, G. (2008b). Modulating activity in the motor cortex affects performance for the two hands differently depending upon which hemisphere is stimulated. Eur. J. Neurosci. 28, 1667-1673. doi: 10.1111/j.1460-9568.2008.06459.x

Vitor-Costa, M., Okuno, N. M., Bortolotti, H., Bertollo, M., Boggio, P. S., Fregni, F., et al. (2015). Improving cycling performance: transcranial direct current stimulation increases time to exhaustion in cycling. PLOS ONE 10:e0144916. doi: 10.1371/journal.pone.0144916

Vollmann, H., Conde, V., Sewerin, S., Taubert, M., Sehm, B., Witte, O. W., et al. (2013). Anodal transcranial direct current stimulation (tDCS) over supplementary motor area (SMA) but not pre-SMA promotes shortterm visuomotor learning. Brain Stimul. 6, 101-107. doi: 10.1016/j.brs.2012. 03.018

Waters, S., Wiestler, T., and Diedrichsen, J. (2017). Cooperation not competition: bihemispheric tDCS and fMRI show role for ipsilateral hemisphere in motor learning. J. Neurosci. 37, 7500-7512. doi: 10.1523/JNEUROSCI.341416.2017

Waters-Metenier, S., Husain, M., Wiestler, T., and Diedrichsen, J. (2014). Bihemispheric transcranial direct current stimulation enhances effectorindependent representations of motor synergy and sequence learning. $J$. Neurosci. 34, 1037-1050. doi: 10.1523/JNEUROSCI.2282-13.2014

Westwood, S. J., and Romani, C. (2017). Transcranial direct current stimulation (tDCS) modulation of picture naming and word reading: a meta-analysis of single session tDCS applied to healthy participants. Neuropsychologia 104, 234-249. doi: 10.1016/j.neuropsychologia.2017.07.031

Williams, J. A., Pascual-Leone, A., and Fregni, F. (2010). Interhemispheric modulation induced by cortical stimulation and motor training. Phys. Ther. 90 398-410. doi: 10.2522/ptj.20090075

Williams, P. S., Hoffman, R. L., and Clark, B. C. (2013). Preliminary evidence that anodal transcranial direct current stimulation enhances time to task failure of a sustained submaximal contraction. PLOS ONE 8:e81418. doi: 10.1371/journal.pone.0081418

Woods, A. J., Antal, A., Bikson, M., Boggio, P. S., Brunoni, A. R., Celnik, P., et al. (2016). A technical guide to tDCS, and related non-invasive brain stimulation tools. Clin. Neurophysiol. 127, 1031-1048. doi: 10.1016/j.clinph.2015. 11.012

Zhu, F. F., Yeung, A. Y., Poolton, J. M., Lee, T. M. C., Leung, G. K. K., and Masters, R. S. W. (2015). Cathodal transcranial direct current stimulation over left dorsolateral prefrontal cortex area promotes implicit motor learning in a golf putting task. Brain Stimul. 8, 784-786. doi: 10.1016/j.brs.2015. 02.005

Zimerman, M., Heise, K. F., Gerloff, C., Cohen, L. G., and Hummel, F. C. (2014). Disrupting the ipsilateral motor cortex interferes with training of a complex motor task in older adults. Cereb. Cortex 24, 1030-1036. doi: 10.1093/cercor/bhs385

Zimerman, M., Nitsch, M., Giraux, P., Gerloff, C., Cohen, L. G., and Hummel, F. C. (2013). Neuroenhancement of the aging brain: restoring skill acquisition in old subjects. Ann. Neurol. 73, 10-15. doi: 10.1002/ana.23761

Conflict of Interest: The authors declare that the research was conducted in the absence of any commercial or financial relationships that could be construed as a potential conflict of interest.

Copyright (c) 2019 Patel, Ashcroft, Patel, Ashrafian, Woods, Singh, Darzi and Leff. This is an open-access article distributed under the terms of the Creative Commons Attribution License (CC BY). The use, distribution or reproduction in other forums is permitted, provided the original author(s) and the copyright owner(s) are credited and that the original publication in this journal is cited, in accordance with accepted academic practice. No use, distribution or reproduction is permitted which does not comply with these terms. 


\section{APPENDIX 1}

\section{Search Strategy}

1. exp transcranial direct current stimulation/

2. (transcranial adj5 electric $\$$ adj5 stimulation).mp. $[\mathrm{mp}=\mathrm{ti}, \mathrm{ab}$, hw, tn, ot, dm, mf, dv, kw, fx, dq, nm, kf, px, rx, ui, sy, tc, id, $\mathrm{tm}$ ]

3. (transcranial adj5 DC adj5 stimulation).mp. [mp=ti, ab, hw, tn, ot, dm, mf, dv, kw, fx, dq, nm, kf, px, rx, ui, sy, tc, id, tm]

4. (transcranial adj5 direct current adj5 stimulation).mp. $[\mathrm{mp}=\mathrm{ti}, \mathrm{ab}, \mathrm{hw}, \mathrm{tn}, \mathrm{ot}, \mathrm{dm}, \mathrm{mf}, \mathrm{dv}, \mathrm{kw}, \mathrm{fx}, \mathrm{dq}, \mathrm{nm}, \mathrm{kf}, \mathrm{px}$, rx, ui, sy, tc, id, tm]

5. tdcs.mp. [mp=ti, ab, hw, tn, ot, dm, mf, dv, kw, fx, dq, nm, kf, px, rx, ui, sy, tc, id, tm]

6. or/1-5

7. Pragmatic Clinical Trial.pt.

8. Randomized Controlled Trial.pt.

9. exp Randomized Controlled Trials as Topic/

10. "Randomized Controlled Trial (topic)"/

11. Randomized Controlled Trial/

12. Randomization/

13. Random Allocation/

14. Double-Blind Method/

15. Double Blind Procedure/

16. Double-Blind Studies/

17. Single-Blind Method/

18. Single Blind Procedure/

19. Single-Blind Studies/

20. Placebos/

21. Placebo/

22. (random $*$ or sham or placebo $*$ ).mp.

23. ((singl $*$ or doubl $*)$ adj (blind $*$ or dumm $*$ or mask $*))$.mp.

24. or $/ 7-23$

25. 6 and 24

26. limit 25 to "all adult (18 plus years)"

27. limit 26 to english language

28. limit 27 to human

29. remove duplicates from 28 